\title{
SARS-CoV-2 membrane protein causes the mitochondrial apoptosis and pulmonary edema via targeting BOK
}

\author{
Yang Yang $\mathbb{D}^{1,2,3,5}$, Yongjian $\mathrm{Wu}^{1,2,3,5}$, Xiaojun Meng ${ }^{1}$, Zhiying Wang ${ }^{1}$, Muhammad Younis ${ }^{1}$, Ye Liu ${ }^{1}$, Peihui Wang ${ }^{4}{ }^{4}$ and
} (c) The Author(s), under exclusive licence to ADMC Associazione Differenziamento e Morte Cellulare 2022

Deaths caused by coronavirus disease 2019 (COVID-19) are largely due to the lungs edema resulting from the disruption of the lung alveolo-capillary barrier, induced by SARS-CoV-2-triggered pulmonary cell apoptosis. However, the molecular mechanism underlying the proapoptotic role of SARS-CoV-2 is still unclear. Here, we revealed that SARS-CoV-2 membrane (M) protein could induce lung epithelial cells mitochondrial apoptosis. Notably, M protein stabilized B-cell lymphoma 2 (BCL-2) ovarian killer (BOK) via inhibiting its ubiquitination and promoted BOK mitochondria translocation. The endodomain of $\mathrm{M}$ protein was required for its interaction with BOK. Knockout of BOK by CRISPR/Cas9 increased cellular resistance to M protein-induced apoptosis. BOK was rescued in the BOK-knockout cells, which led to apoptosis induced by M protein. M protein induced BOK to trigger apoptosis in the absence of $\mathrm{BAX}$ and $\mathrm{BAK}$. Furthermore, the $\mathrm{BH} 2$ domain of $\mathrm{BOK}$ was required for interaction with $\mathrm{M}$ protein and proapoptosis. In vivo $M$ protein recombinant lentivirus infection induced caspase-associated apoptosis and increased alveolar-capillary permeability in the mouse lungs. BOK knockdown improved the lung edema due to lentivirus-M protein infection. Overall, M protein activated the BOK-dependent apoptotic pathway and thus exacerbated SARS-CoV-2 associated lung injury in vivo. These findings proposed a proapoptotic role for M protein in SARS-CoV-2 pathogenesis, which may provide potential targets for COVID-19 treatments.

Cell Death \& Differentiation (2022) 29:1395-1408; https://doi.org/10.1038/s41418-022-00928-x

\section{INTRODUCTION}

Coronavirus disease 2019 (COVID-19) is an acute pneumonic disease caused by severe acute respiratory syndrome coronavirus 2 (SARS-CoV-2), resulting in millions of deaths. Hypoxemia respiratory failure is a characteristic of COVID-19 death, which exhibits abundant cell apoptosis and fluid accumulation in intraalveolar spaces [1, 2]. However, the mechanism of pulmonary barrier failure caused by SARS-CoV-2 infection remains unclear.

It has been proposed that SARS-CoV-2 infection-associated epithelial cell apoptosis plays a vital role in lungs disorder [3]. Apoptosis is evolutionarily conserved progress and has distinctive signal transduction, different from necroptosis and pyroptosis [4]. Apoptosis is the most widely studied form of cell death in the context of virus infections [5]. Studies revealed that coronavirus, such as SARS and MERS, majorly induce apoptosis in target cells, resulting in organ dysfunction and disease [6, 7]. Clinical pathology results indicate that endothelial apoptosis exists in postmortem lung slices from COVID-19 patients [8]. It is reported that SARS-CoV-2 induces the apoptosis of the infected alveolar cells by using human lung stem cell-based alveolospheres [9]. In humanized ACE2 transgenic mice and Syrian hamsters, extensive apoptosis has been observed in lung epithelial cells [10-12]. However, the mechanism of apoptosis on pulmonary epithelial cells induced by SARS-CoV-2 remains unclear.

Apoptosis can be modulated by an interaction between cellular and viral proteins [6]. Coronavirus, such as SARS-CoV and SARSCoV-2, is a kind of enveloped virus, including four structural proteins: spike $(\mathrm{S})$, nucleocapsid $(\mathrm{N})$, membrane $(\mathrm{M})$ and envelope (E) protein, some non-structural proteins and putative accessory factors [13]. Among the SARS-CoV proteins, M protein, E protein, $3 a, 7 a$ protein can induce cell apoptosis $[6,14,15]$. However, the potential viral protein and molecular mechanism involved in SARS-CoV-2-induced apoptosis need more investigation.

Proteomic analysis of host cells infected by SARS-CoV-2 indicated that mitochondria is a primary target organelle. Moreover, many mitochondrial apoptosis mediators, like cytochrome c (Cyt c) are upregulated in SARS-CoV-2-infected cells [16]. Mitochondrial apoptosis is regulated by the B-cell lymphoma-2 (BCL-2) family, consisting of pro-apoptotic and anti-apoptotic members. Once activated, the pro-apoptotic effectors, like BCL-2 ovarian killer protein (BOK), will translocate to mitochondria and perforate it, which induces apoptosis through releasing signaling factors such as Cyt c [17].

In this study, we found that M protein incited the BOK level by interacting and inhibiting its ubiquitination-degradation. The

\footnotetext{
${ }^{1}$ Center for Infection and Immunity and Guangdong Provincial Key Laboratory of Biomedical Imaging, The Fifth Affiliated Hospital of Sun Yat-sen University, Zhuhai, Guangdong Province 519000, China. ${ }^{2}$ The Sixth Affiliated Hospital of Guangzhou Medical University, Qingyuan People's Hospital, Qingyuan, Guangdong Province 511518, China. ${ }^{3}$ Key

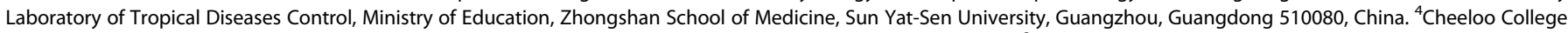

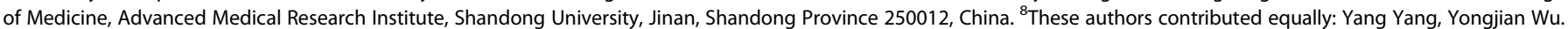

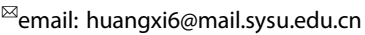
Edited by DL Vaux
}

Received: 20 June 2021 Revised: 18 December 2021 Accepted: 28 December 2021 Published online: 12 January 2022 

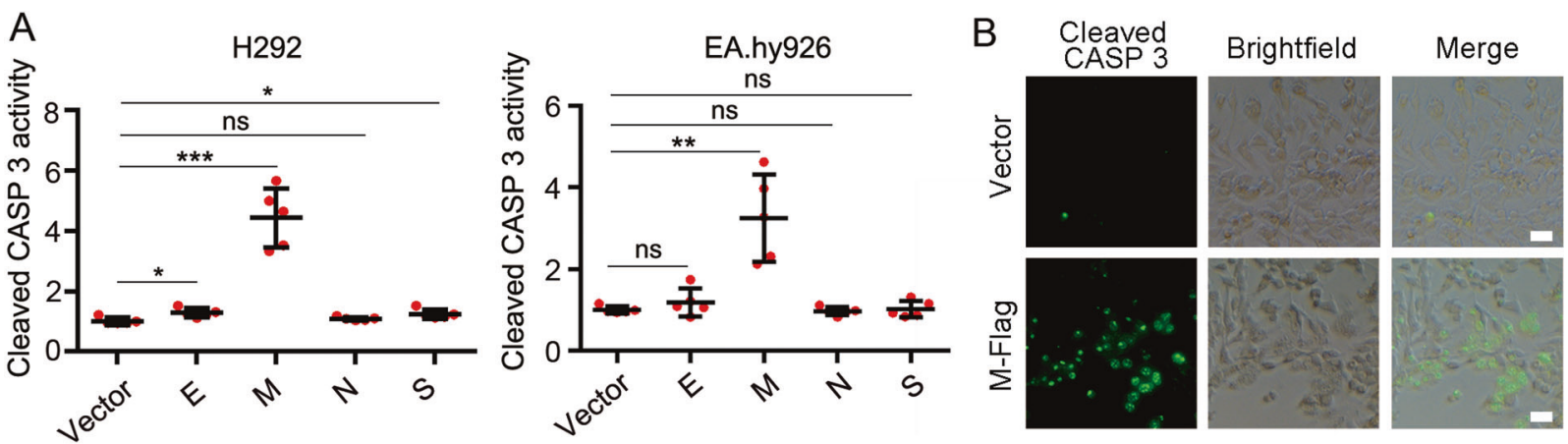

C

$\mathrm{H} 292$
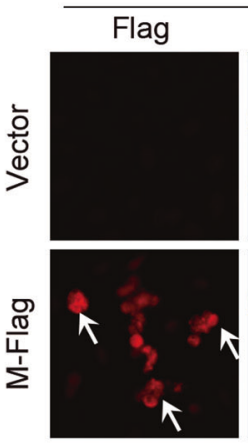

$\mathrm{D}$
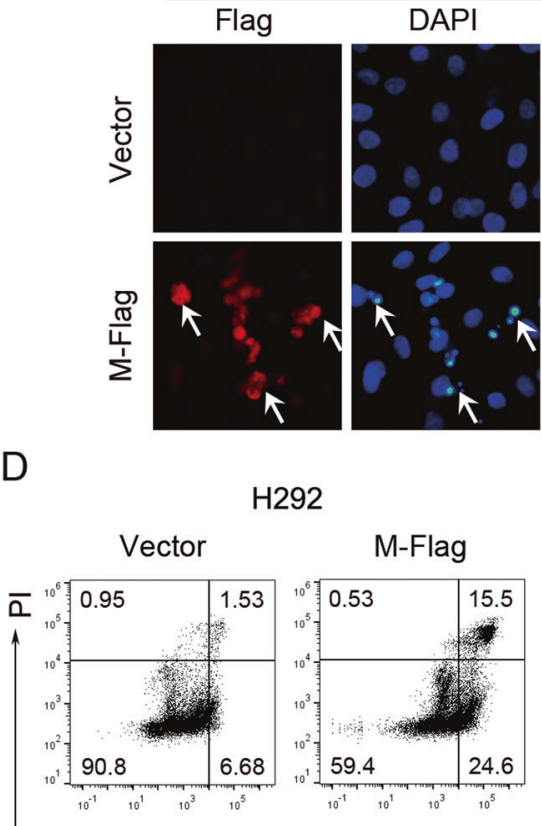
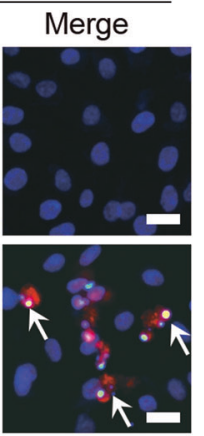

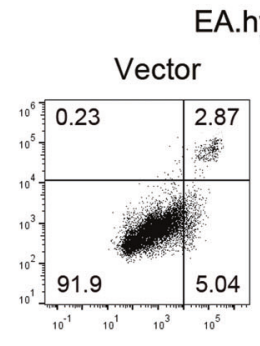

EA.hy926

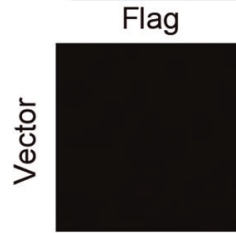

EA.hy926
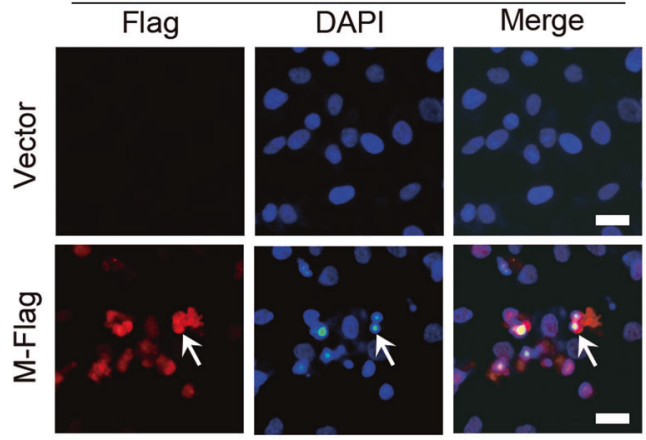

E

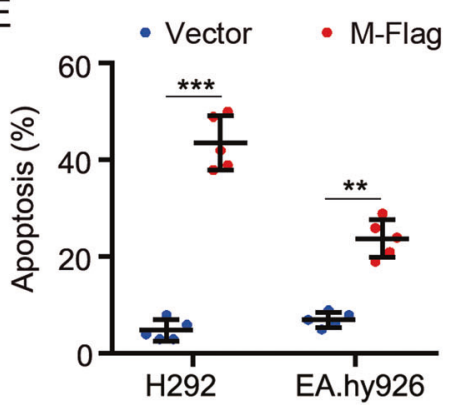

$\mathrm{F}$

H292 transfected with M-Flag plasmid
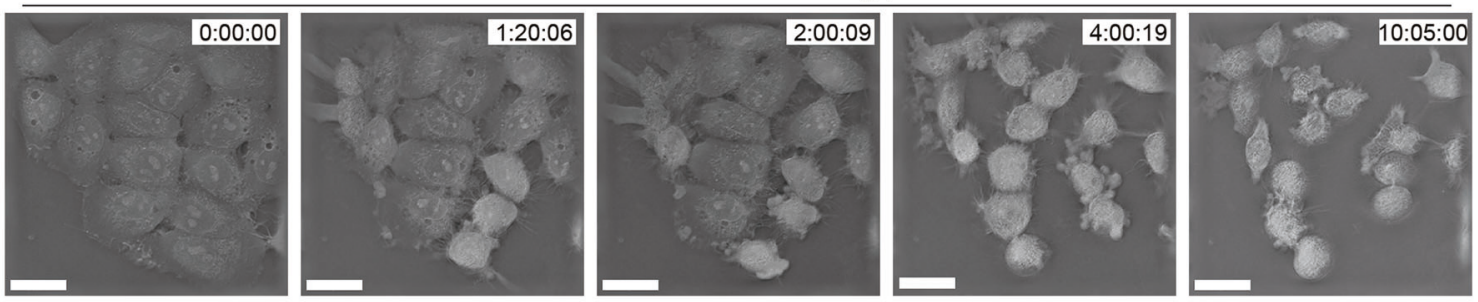

EA.hy926 transfected with M-Flag plasmid
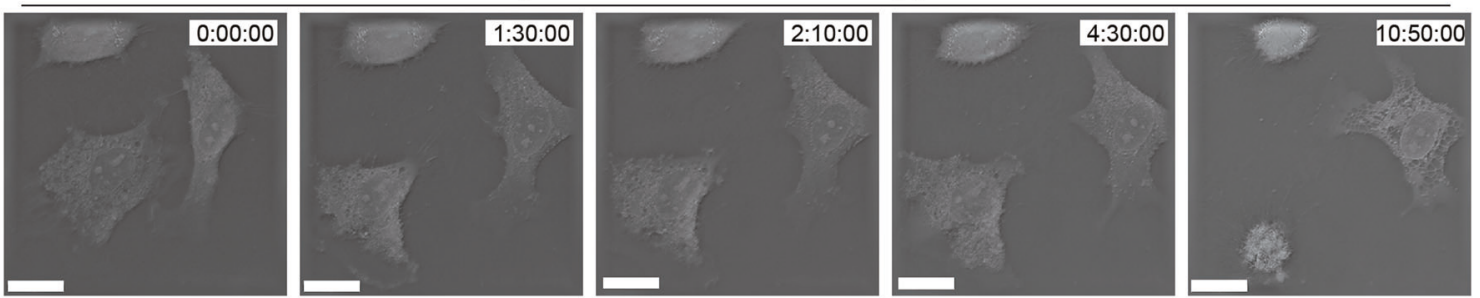

endodomain of $M$ protein was required for its interaction with $\mathrm{BOK}$. Furthermore, the $\mathrm{BH} 2$ domain of $\mathrm{BOK}$ was essential for the $\mathrm{M}$ protein/BOK interaction and mitochondrial apoptosis. In vivo results suggested that $M$ protein mediated apoptosis and caused remarkable lung edema in the mice model. BOK knockdown alleviated the lung edema due to $M$ protein. Overall, our study revealed the role of $M$ protein in SARS-CoV-2-induced lung cell apoptosis by triggering BOK-dependent mitochondrial death and might provide targeting interventions for the treatment of the COVID-19 lung disorders. 
Fig. 1 The impacts of SARS-CoV-2 M protein overexpression on $\mathbf{H 2 9 2}$ and EA.hy926 cell apoptosis. A Cleaved caspase 3 (cleaved CASP 3 ) activity in $\mathrm{H} 292$ and EA.hy926 cells was measured after $48 \mathrm{~h}$ transfection with an empty vector (pcDNA6B), or plasmids expressing SARS-CoV-2 E protein, M protein, N and S protein, respectively. B Represent images of $\mathrm{H} 292$ cells staining with cleaved CASP 3 detection reagent. Scale bar, $50 \mu \mathrm{m}$. C H292 and EA.hy926 cells were transfected with an empty vector (pcDNA6B) or a M-Flag expression vector for $48 \mathrm{~h}$. Representative multiplexed immunofluorescence staining of DAPI (blue) and antibody-Flag (red) were showed. Scale bar, $20 \mu \mathrm{m}$. D, E Flow cytometric analysis was used for measuring the proportion of apoptotic cells stained with annexin V-fluorescein 5-isothiocyanate (FITC)/propidium iodide (PI) (D) and the proportion of apoptotic cells was further analyzed (E). F Live-cell images of H292 and EA.hy926 transfected with M plasmid. Scale bar: $20 \mu \mathrm{m}$. See also Movies S1 and S2. Data present with mean \pm SD for five independent experiments. ${ }^{*} p<0.05,{ }^{* *} p<0.01,{ }^{* * *} p<0.001$, ns nonsignificant. Unpaired Student's t test compared two subjects.

\section{MATERIALS AND METHODS \\ Plasmid construction}

The CDNA encoding BOK was obtained by reverse transcription of total RNA from H292 cell lines and following PCR. The purified PCR products were directionally cloned into pcDNA3.1 (+) ( $\mathrm{HA}$ tag or Myc tag) and pCDHCMV-MCS-EF1-green fluorescent protein (GFP)-T2A-Puro vector (Generay, Shanghai, China). The primers used in this study were shown in Supplementary Table 1. The pcDNA6B-nCoV-M-flag, pcDNA6B-nCoV-M$\Delta$ transmembrane (TM)1-flag, pcDNA6B-nCoV-M- $\triangle \mathrm{TM} 12$-flag, and pcDNA6B-nCoV-M- $\triangle$ TM1-3-flag, pcDNA6B-nCoV-M- $\Delta$ endodomain (ED)-flag clone were kind gifts from Prof. Wang Peihui $[18,19]$. HA-tagged BOK plasmids were lacking $\mathrm{BH} 4$, lacking $\mathrm{BH} 3$, lacking $\mathrm{BH}$, lacking $\mathrm{BH} 2$ or lacking transmembrane evolved from full length (FL) of BOK, pLKO.1shRNA (shCtrl.), pLKO.1-shRNA-mouse BOK (mBOK)-1 (Lenti-shBOK1) and pLKO.1-shRNA-mBOK2 (shBOK2) construct were purchased from Generay company (Shanghai, China). The shRNA sequences for BOK knockdown were listed in Supplementary Table 1.

\section{Transfection}

The indicated vectors were transfected with Lipofectamine 2000 (Thermo Fisher Scientific, Waltham, MA, USA) or polyethyleneimine (linear MW25,000, Fushen, Shanghai, China) following the manufacturer's protocol. If the time point was not indicated specially, otherwise cells were harvested $48 \mathrm{~h}$ post transfection and processed for Western blot (WB) or flow cytometric analysis.

\section{Mitochondrial membrane potential $(\Delta \Psi \mathrm{m})$ assay}

$\Delta \psi_{\mathrm{m}}$ variations were detected by JC-1 staining (Beyotime, China). Briefly, $2 \times 10^{4}$ cells were seeded in 24-well plate (Corning, Carolina, USA) overnight. JC-1 staining was performed after $24 \mathrm{~h}$ of transfection with 1 $\mu \mathrm{g}$ of mock or M-flag plasmid per-well, with $10 \mathrm{nM} \mathrm{JC}-1$, according to the manufacturer's instructions. Then cells were washed with PBS and observed by inverted fluorescence microscopy (Zeiss LSM 40, Carl Zeiss, Germany). Also, we recorded the ratio of aggregated JC-1 (red fluorescence; ECD channel) to monomeric JC-1 (green fluorescence, FITC channel) with flow cytometry. The reduction of $\Delta \Psi_{\mathrm{m}}$ was indicated by the $\mathrm{red} / \mathrm{green}$ fluorescence intensity ratio.

\section{Cell lines}

NCL-H292 [H292] (\#SCSP-582), HEK293T (\#SCSP-502), EA.hy926 (\#GNHu39) and Lewis lung cancer (LLC, \#TCM 7) cell lines were purchased from the National Collection of Authenticated Cell Cultures (Shanghai, China). All the cells were cultured in a humidified atmosphere of $5 \% \mathrm{CO}_{2}$ at $37^{\circ} \mathrm{C} . \mathrm{H} 292$ cells were grown in RPMI-1640 (Gibico, New York, USA) medium supplemented with $10 \%$ fetal bovine serum (FBS) (HyClone, Utah, USA) and $100 \mathrm{U} / \mathrm{ml}$ Penicillin-Streptomycin (Gibico, USA). HEK293T, EA.hy926 and LLC cells were cultured in Dulbecco's modified Eagle's medium (Gibco, USA) supplemented with $10 \%$ FBS and $100 \mathrm{U} / \mathrm{ml}$ Penicillin-Streptomycin.

\section{Apoptosis assay}

Apoptosis assay was carried out by Annexin V-FITC/PI Detection Kit (BD, San Diego, CA, USA) followed by the manufacturer's instruction. Briefly, $2 \times$ $10^{5}$ cells were resuspended in binding buffer, followed by staining with 10 $\mu \mathrm{l}$ Annexin V-FITC and $5 \mu \mathrm{l} \mathrm{PI}$ for $15 \mathrm{~min}$. The samples were analyzed with flow cytometry (Beckman Coulter, cytoflexLX, USA).

\section{Animals}

All animal studies were approved by the Ethics Committee of Sun-Yat Sen University (No.00109), in accordance with UK Animals (Scientific Procedures) Act 1986. C57BL/6 mice were bred in a 12-h light/dark cycle with access freely to water and food specific pathogen-free facility. Sex- and age-
(6-8 weeks-old) matched mice were randomly assigned into 8 groups: the Control group, Lenti-vector group (lentiviral negative control vector encoding mcherry reporter gene), Lenti-M group (lentiviral vector encoding $M$ protein and mcherry reporter gene), Lenti-M + Q-VD-OPh group, PBS + Lenti-M group (PBS group), Lenti-shcontrol + Lenti-M (shCtrl.) group, Lenti-shBOK1 + Lenti-M (shBOK1) group, Lenti-shBOK2 + Lenti-M (shBOK2) group.

$5 \times 10^{7} \mathrm{TU} / \mathrm{kg}$ in $50 \mu \mathrm{l}$ PBS of the lentivirus (Lenti-vector, Lenti-M, LentishControl, Lenti-shBOK1, and Lenti-shBOK2) or $50 \mu \mathrm{l} \mathrm{PBS}$ were intratracheally injected. For knockdown animal models, $5 \times 10^{7} \mathrm{TU} / \mathrm{kg}$ in $50 \mu \mathrm{l}$ PBS of the lentivirus expressing $M$ protein was intratracheally injected after three days of the injection of a lentivirus expressing shRNA. Before intratracheal injection, the mice were treated with pentobarbital sodium $(80 \mathrm{mg} / \mathrm{kg})$. $26 \mathrm{G}$ syringes were used for injection of surgical exposure bronchus. Before sacrifice, the mouse lungs were quantified by computed tomography (CT) using a dedicated small animal PET-CT scanner (nanoScan PET/CT 82 s, Mediso, Arlington, USA). Images were analyzed by RadiAnt DICOM Viewer software (version 2020.1).

\section{Bioluminescence imaging}

The thorax was surgically exposed and the lungs were washed with PBS. The bioluminescent images of the lung organ were taken using an IVIS ${ }^{\circledR}$ Lumina III (PerkinElmerzei, USA).

\section{Bronchoalveolar lavage fluid (BALF)}

After euthanasia of mice with ketamine/xylazine, the collection of bronchoalveolar lavage fluid (BALF) was followed according to the previous study [20]. The trachea was semi-exposed and the lungs were washed with $0.8 \mathrm{ml}$ PBS three times. The right lung lobe was grinded and lysis buffer was added for WB analysis and the left lobe was used for Haemotoxinlin and Eosin (H\&E) and immunofluorescence staining assays. The BALF supernatant was centrifuged at $550 \mathrm{~g}$ for $5 \mathrm{~min}$ at $4{ }^{\circ} \mathrm{C}$. Cells were resuspended with $500 \mu \mathrm{l}$ PBS and counted using a hemocytometer. The supernatant was stored at $-80^{\circ} \mathrm{C}$ for further experiments.

For quantification of BALF proteins, the BALF supernatant was measured with Coomassie Brilliant Blue G assay. For the IgM measurement, the BALF supernatant was tested with a Mouse IgM ELISA development kit (MABTECH, 3885-1AD-6, Sweden) as per the manufacturer's instructions. Other animals were used for pulmonary permeability assay.

\section{Pulmonary permeability assay}

Pulmonary Evans blue (EB) extravasation was measured as previously reported method [21]. Briefly, mice were intravenously infused with EB $(30 \mathrm{mg} / \mathrm{kg}) 60$ min prior to being sacrificed. The left lungs were excised and stored in liquid nitrogen. Frozen tissue was incubated individually in formamide $\left(60^{\circ} \mathrm{C}, 36 \mathrm{~h}\right)$. The quantity of EB extracted from the tissue was evaluated using the ELISA reader (Thermo Fisher Scientific, USA) at the absorption maximum for EB (620 $\mathrm{nm})$. Lungs were weighed after being dried $65^{\circ} \mathrm{C}$ for $48 \mathrm{~h}$. The amount of $E B$ was calculated following the standard curve of $\mathrm{EB}$ in formamide/PBS. Tissue EB content $=$ Lung EB content/dry tissue weight.

\section{Caspase (CASP) activity assay}

CASP 3 activity in living cells was tested with the GreenNuc ${ }^{\text {TM }}$ CASP -3 Activity Kit (Beyotime, China) according to the manufacturer's instructions. H292 or EA.hy926 $\left(2 \times 10^{4}\right.$ cells per well) cultured in 96 -well plates were transfected with $0.5 \mu \mathrm{g}$ of empty plasmid or plasmids expressing S-flag, $\mathrm{N}$ flag, M-flag or $\mathrm{N}$-flag, respectively, for $48 \mathrm{~h}$. Then, cells were incubated with $200 \mu \mathrm{l}$ PBS containing $10 \mu \mathrm{M}$ active CASP 3 detection reagents for $30 \mathrm{~min}$ at room temperature. The fluorescent intensity of CASP 3 activity was quantified using the ELISA reader (Thermo Fisher Scientific, USA) at the absorption of $485 \mathrm{~nm}$ (excitation wavelength).

CASP 8 (Beyotime, China), CASP 9 (Beyotime, China), and CASP 12 (SLF Biotech, China) activities were measured using the CASP Activity Kit according 
A

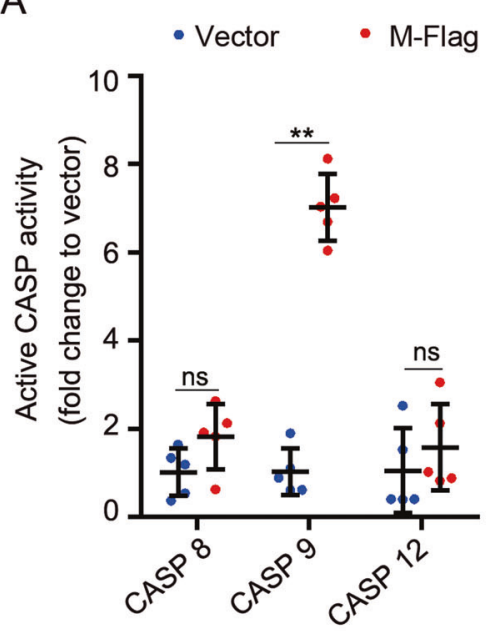

B

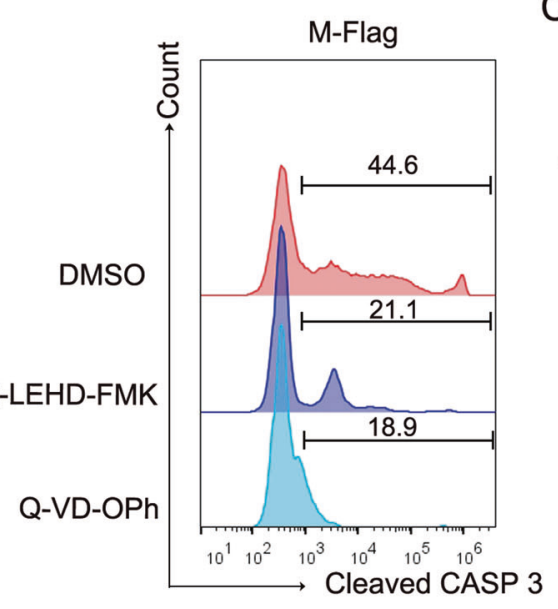

C

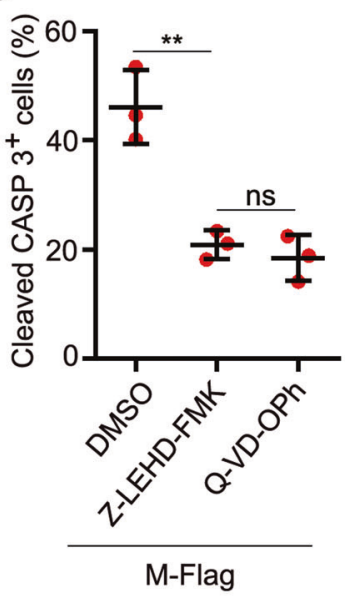

D

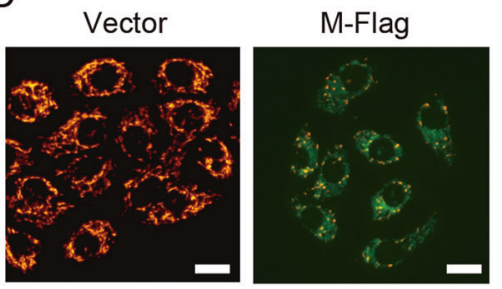

JC-1 aggregates/JC-1monomer

G
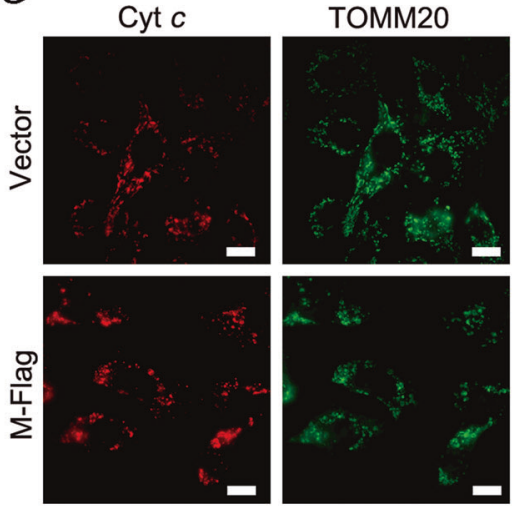

E
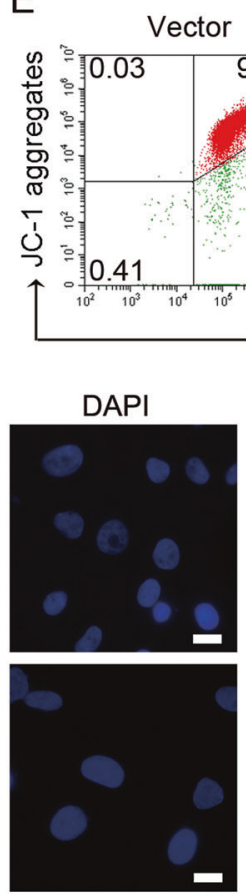

ector
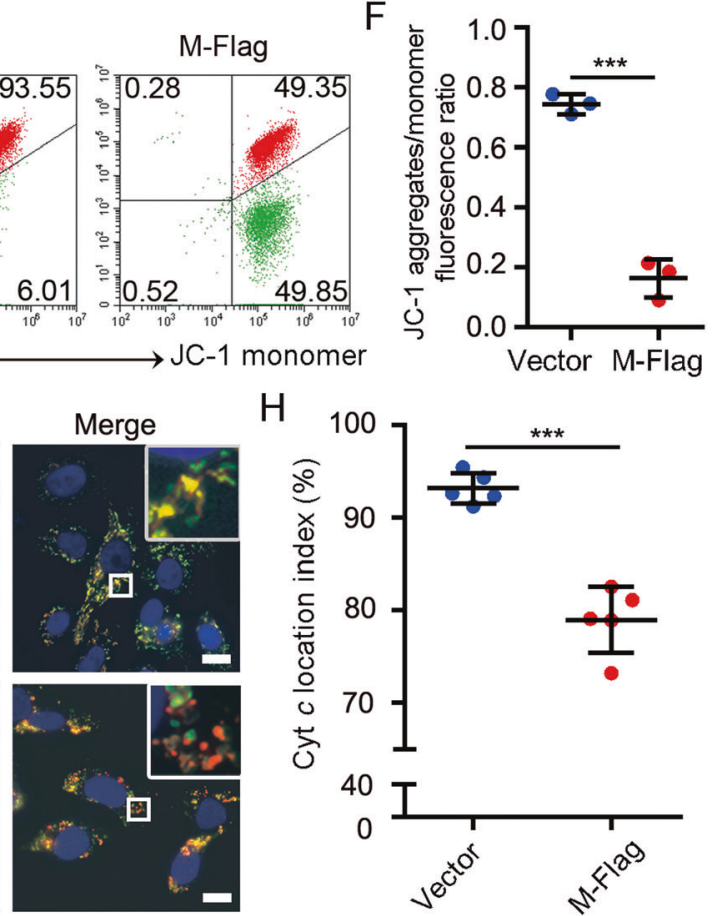

$\mathrm{H}$

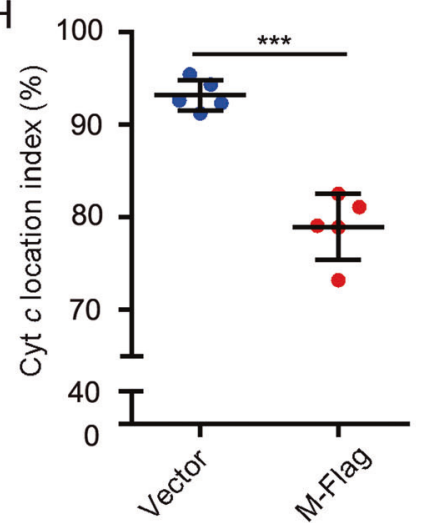

Fig. 2 SARS-CoV-2 M protein induced mitochondrial death in $\mathbf{H 2 9 2}$ cells. H292 cells were transfected with an empty vector (pcDNA6B) or M-Flag plasmid and used for further experiments. A Activities of CASP 8, CASP 9 and CASP 12 were examined at $48 \mathrm{~h}$. Data are representative of three independent experiments performed in triplicates. B, C The proportion of cells displaying cleaved CASP $3^{+}$activation was assayed with FACS in H292 cells transfected with M-Flag for $48 \mathrm{~h}$ in the presence of Z-LEHD-FMK (CASP 9 inhibitor, $20 \mu \mathrm{M}$ ) or Q-VD-OPh (pan CASP inhibitor, $50 \mu \mathrm{M})$. D Representative live images of JC-1 fluorescence in $\mathrm{H} 292$ cells as an indicator of mitochondrial membrane potential ( $\Delta \Psi \mathrm{m}$ ). Low $\Delta \Psi \mathrm{m}$ (accumulation of JC-1 monomers) demonstrated with green, normally polarized mitochondrial membranes (accumulation of JC-1 aggregates) presented orange. The images were acquired using a Zeiss 880 inverted confocal microscope. Scale bar, $50 \mu \mathrm{m}$. E, F The JC-1 signal was measured by flow cytometry. The red to green JC-1 fluorescence ratio in $\mathrm{H} 292$ cells was quantified by flowjo software (F). G Represent images of $\mathrm{H} 292$ cells stained with Cyt $c$ and TOMM20 (mitochondria marker) at $36 \mathrm{~h}$. The images were acquired using a Zeiss 880 inverted confocal microscope with a $63 \times$ oil-immersion objective. Scale bar, $50 \mu \mathrm{m}$. H Co-localization of mitochondria (TOMM20, green) and Cyt $c$ (red) was analyzed. $n=5-10$ images $/ 63 \times$ fields experiment. Data present with mean \pm SD for at least three independent experiments. $\mathrm{ns}$ non-significant, ${ }^{*} p<0.05,{ }^{* *} p<0.01,{ }^{* * *} p<0.001$. Unpaired Student's t test compared two subjects.

to the manufacturer's instructions. Briefly, transfected ( $2 \mu \mathrm{g}$ of mock or M-flag) of $\mathrm{H} 292\left(1 \times 10^{6}\right.$ well) were scraped and lysates were left on ice for $5 \mathrm{~min}$. The buffer containing the substrate peptides coupling p-nitroanilide (pNA) was added to the supernatant. The signal of pNA or CASP activity was detected at the wavelength of $405 \mathrm{~nm}$ by an ELISA reader (Thermo Fisher Scientific, USA).

\section{Generation of BOK knockout cell lines and rescue expression of BOK}

LentiCRISPR v2-derived constructs (Addgene \#52961) encoding sgRNA targeted BAK, BAX or BOK and Cas9 were cotransfected with psPAX2
(Addgene \#12260) and pMD2.G (Addgene \#12259) into 293 T cells at a 10:7:5 (a total of $16 \mu \mathrm{g}$ plasmids) ratio on a $10 \mathrm{~cm}$ plate with polyethyleneimine. The supernatant of the culture medium was collected $48 \mathrm{~h}$ post transfection and filtered through a $0.45 \mu \mathrm{m}$ filter. $\mathrm{H} 292$ cells were transducted with lentivirus in the presence of $6 \mu \mathrm{g} / \mathrm{ml}$ polybrene (Santa Cruz Bio., Texas, USA) for $12 \mathrm{~h}$. Three days after transduction, cells were diluted into single cell per well and were selected with $2 \mu \mathrm{g} / \mathrm{ml}$ puromycin (Sigma-Aldrich, St. Louis, Missouri, USA). Knockout status of expanded single-cell clones was validated by gene sequences and WB analysis of immunoblotting with BAK antibody (1:1000, \#ab32371, Abcam, Cambridge, 
A

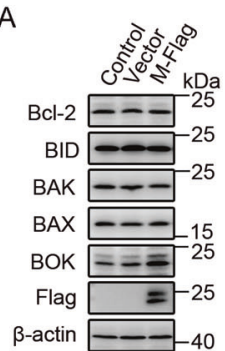

$\mathrm{E}$

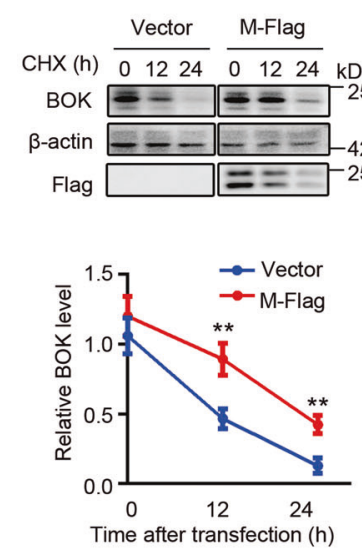

$\mathrm{H}$

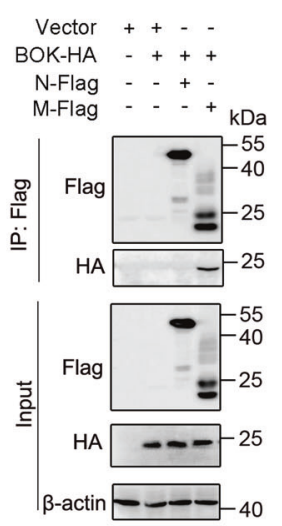

K
B

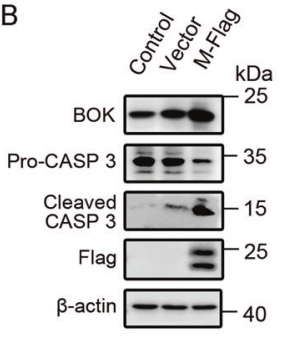

$\mathrm{F}$
C

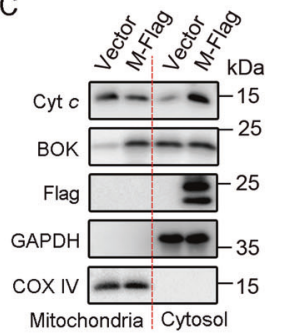

D

G

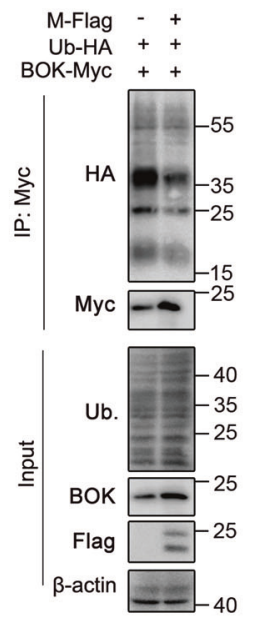

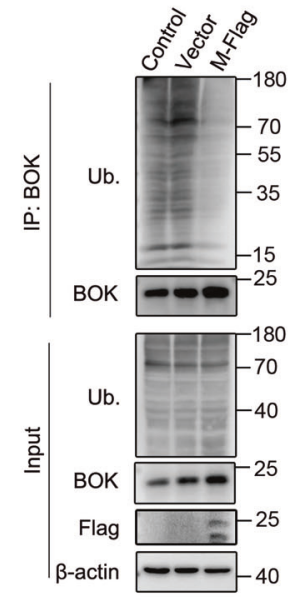

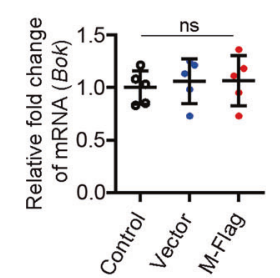

Vector $+++-\quad J$ BOK-HA - + +

M-Flag - + +
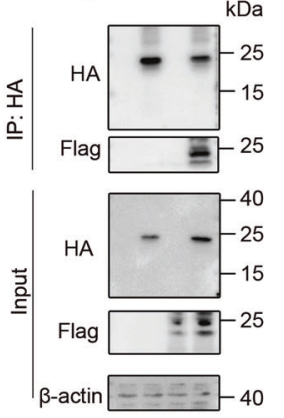

J Vector + . . . -

M-Flag -

M- $\triangle$ TM1-Flag -

M- $\triangle$ TM1-2-Flag -

M- $\triangle$ ED-Flag - - - - +

BOK-HA +++++ kDa
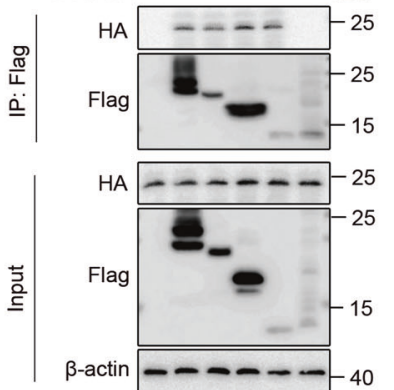

L

M- $\triangle$ TM1-Flag

$\begin{array}{lllll}\text { Vector + } & - & - & - & - \\ \text { M-Flag - }+ & - & - & - & -\end{array}$

M- $\triangle$ TM1-3-Flag - - - +

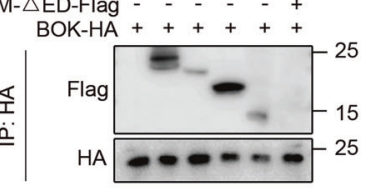

Flag(M)

$\mathrm{HA}(\mathrm{BOK})$
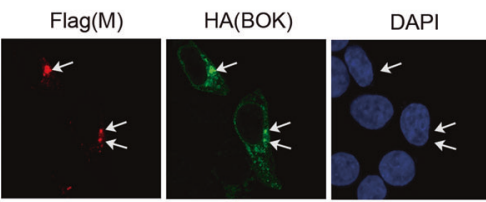

Merge
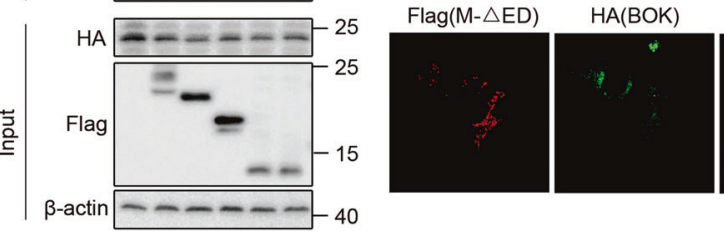

DAPI

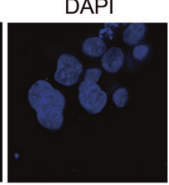

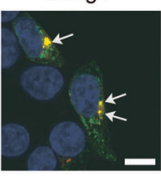

Merge

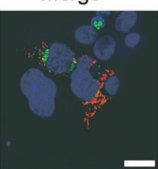

England), BAX antibody (1:1000, \#2772 s, CST, USA) or BOK antibody (1:1000, \#ab233072, Abcam, Cambridge, England). The rescue expression of BOK followed Xu et al. [22]. The sgRNA sequences for CRISPR/Cas 9 knockout were listed in Supplementary Table 1.
Lentivirus packaging and infection

The $\mathrm{pCDH}$-CMV-MCS-EF1-GFP-T2A-Puro encoding BOK or pLVX-mcherry vector encoding SARS-CoV-2 M protein or pLKO.1 vector encoding mouseBOK (mBok)-shRNA was cotransfected with psPAX2 and pMD2G into 293 
Fig. 3 SARS-CoV-2 $M$ protein inhibited BOK ubiquitination and promoted its translocation and interacted with BOK via endodomain. A, B $\mathrm{H} 292$ cells were not transfected (control) or transfected with an empty vector (pcDNA6B) or M-Flag plasmid for $48 \mathrm{~h}$. The protein levels of Bcl2, BID, BAX, BAK and BOK (A) or M-Flag, Precursor caspase-3 (Pro-CASP 3) and cleaved CASP 3 were analyzed by WB. C After H292 cells were transfected with M-Flag for $48 \mathrm{~h}$, the mitochondrial and cytosolic fractions were isolated and analyzed by WB. D mRNA levels of Bok in H292 cells transfection with M-Flag for $48 \mathrm{~h}$ were examined by qPCR. E Cycloheximide (CHX) chase to check the turnover of endogenous BOK, H292 cells either transfected with vector or M-Flag expressing plasmid, were treated with $30 \mu \mathrm{g} / \mathrm{ml} \mathrm{CHX}$ and harvested at the indicated time periods for WB analysis (upper panel), and quantification of BOK level. $\beta$-actin use as internal control (lower panel). F Lysates from H292 nontransfected (control) or transfected with an empty vector (pcDNA6B) or M-Flag plasmid followed by treatment with $10 \mu \mathrm{M}$ MG132 for $5 \mathrm{~h}$ before harvest and were immunoprecipitated. Ub Ubiquitination. G HEK293T cells were cotransfected with plasmids encoding HA-tagged ubiquitin, Myc-tagged BOK, together with either an empty vector (pcDNA3.1(+)) or plasmid encoding Flag-tagged M protein for $40 \mathrm{~h}$. Cells were treated with $10 \mu \mathrm{M}$ MG132 for $5 \mathrm{~h}$ and lysed. Then the proteins mixture was immunoprecipitated with anti-Myc antibody followed by WB. H Immunoprecipitation using anti-Flag or anti-HA antibodies from lysates of 293 T cells transfected with HA-tagged BOK (24 kDa) alone, or with an empty vector (pcDNA6B), SARS-CoV-2 Flag-tagged N (51 kDa) and M (23/24 kDa). I Exogenous protein interaction was further confirmed in 293 T lysates immunoprecipitated with anti-HA and analyzed by WB with the indicated antibodies. J, K $293 \mathrm{~T}$ cells were cotransfected with pcDNA3.1 (+) carrying HA-tagged BOK, with a pcDNA6B vector containing Flag-tagged M (full lengh), Flag-tagged truncated variants of $M$ proteins (M- $\Delta$ transmembrane (TM)1-Flag (22 kDa), M- $\triangle T M 1-2-F l a g ~(18 / 20 \mathrm{kDa}), \mathrm{M}-\Delta T M 1-3-F l a g ~(14 \mathrm{kDa})$, M- $\Delta$ endodomain (ED)Flag (22 kDa)). Blots of cell lysates (input) or anti-Flag (J) and anti-HA (K) immunoprecipitates were analyzed by Western blotting for HA and Flag. L Confocal microscopy of 293 T cells co-transfected with HA-tagged BOK and Flag-tagged M (above lane) or Flag-tagged M- $\Delta \mathrm{ED}$ (below lane). White arrows represent the colocalization. Scale bars, $10 \mu \mathrm{m}$. Data present with mean \pm SD for at least three independent experiments. ns non-significant, ${ }^{* *} p<0.01$. Data were analyzed with one-way ANOVA with LSD't test.

\section{4}

T cells as mentioned above. Culture supernatants were collected $48 \mathrm{~h}$ of post transfection and then centrifuged at $4000 \mathrm{~g}$ for $15 \mathrm{~min}$. Supernatants containing lentiviral particles were concentrated by polyethylene glycol 8000 (PEG 8000) solution at $4{ }^{\circ} \mathrm{C}$ overnight, and then concentrated by centrifugation at $4000 \times g$ for $30 \mathrm{~min}$. The sediments were resuspended in PBS and the virus titer was measured by fluorescence tittering assay or real-time quantitative PCR (qPCR).

\section{Cell fractionation}

Mitochondria and cytosol were separated according to the manufacturer's instruction (Mitochondria Isolation Kit, Thermo Fisher Scientific, USA). Briefly, cells were scraped into Reagent buffer, incubated $8 \mathrm{~min}$ on ice, dounce homogenized. The lysate was centrifuged at $700 \mathrm{~g}$ for $10 \mathrm{~min}$ at $4^{\circ}$ C. After that, the supernatant was collected and centrifuged at $12,000 \mathrm{~g}$ for $15 \mathrm{~min}$ at $4^{\circ} \mathrm{C}$. The pellet contains mitochondrial fraction and the cytosolic fraction was the cytosolic fraction.

\section{Immunoblotting and immunoprecipitation}

Cells pellets or whole grinding animal lung tissue were collected and resuspended in lysis buffer $(10 \mathrm{mM}$ Tris- $\mathrm{HCl}$ [pH7.5], $150 \mathrm{mM} \mathrm{NaCl}, 0.1 \%$ Nonidet p-40, $5 \mathrm{mM}$ EDTA) with PMSF, incubated on ice for $10 \mathrm{~min}$, and centrifuged at $20,000 \times g$ for $15 \mathrm{~min}$. The protein content of cell extracts was quantitated by Coomassie brilliant blue G250 method (Bio-Rad, \#5000205, CA, USA) and boiled at $100^{\circ} \mathrm{C}$ for $5 \mathrm{~min} .30 \mu \mathrm{g}$ total proteins were electrophoresed in 15\% SDS-polyacrylamide gel electrophoresis (PAGE) gel and electro-blotted on to a polyvinylidene difluoride (PVDF) membrane (Millipore, MA, USA). Blots membrane was blocked with $5 \%$ skim milk in TBS with $0.1 \%$ Tween 20 before being incubated with the antibody. The membranes were developed by chemiluminescence (Fdbio, Hangzhou, China) on a Luminescent Image Analyzer (ImageQuant LAS 500, GE, USA).

Immunoprecipitation was carried out as described previously [23]. Briefly, cell extracts were incubated with the anti-Flag affinity gel (\#A2220, Sigma-Aldrich, USA) or the anti-HA agarose beads (\#AT0079, CMCTAG, USA) or suitable primary antibody $(2 \mu \mathrm{g})$ and Protein $\mathrm{A} / \mathrm{G}$ (Millipore, USA) at $4{ }^{\circ} \mathrm{C}$ for $10 \mathrm{~h}$. Next, the mixture was centrifuged at $9500 \mathrm{~g}$ at $4{ }^{\circ} \mathrm{C}$ for $1 \mathrm{~min}$. Pellets were washed eight times with lysis buffer mentioned before. Binding proteins were resuspended in the lysis buffer with the protein loading buffer. Protein samples were subjected to $10 \%$ or $15 \%$ SDS-PAGE gel followed by WB analysis and visualized as mentioned above.

\section{qPCR}

Total RNA was extracted with TRIzol reagent (Life Technologies, USA). 1 $\mu \mathrm{g}$ CDNA was synthesized with M-MLV reverse transcriptase (Promega, \#M1705, Madison, WI, USA) according to the manufacturer's instructions. qPCR reaction was performed using SYBR Green Master Mix (Promega, \#M1705, USA) on a Real-time PCR detection device (CFX Connect ${ }^{\mathrm{TM}}$, BioRad, USA). The primer sequences for human BOK were listed in Supplementary Table 1.

\section{Cycloheximide (CHX) chase assay}

Transfected ( $2 \mu \mathrm{g}$ of empty vector or M-flag) $\mathrm{H} 292$ cells in a 6 well plate and incubated for $24 \mathrm{~h}$. Next, cycloheximide (\#M4879, Abmole, USA) was added to a final concentration of $25 \mu \mathrm{g} / \mathrm{ml}$, and cells were collected at indicated time points. Protein concentration was tested by Quick Start ${ }^{\mathrm{TM}}$ Bradford 1x dye reagent (Bio-Rad, USA) method. WB was performed as described earlier.

\section{In vivo ubiquitination assay}

HEK293T cells or H292 cells were transfected with the indicated plasmids. After treatment with $10 \mu \mathrm{M} \mathrm{MG132}$ (\#M1902, Abmole, USA) for $5 \mathrm{~h}$, the cells were lysed and the supernatant were precipitated and analyzed by Western blotting using appropriate antibodies (including BOK (\#EPR15331, 1:1000, Abcam, USA), Myc, ubiquitin (\#ab7780, 1:1000, Arigobio, Taiwan, China), HA (1:1000, HA.C5, Arigobio, China)) as described previously [23].

\section{Immunofluorescence staining}

Cells were washed with PBS buffer twice and fixed with 4\% paraformaldehyde for $30 \mathrm{~min}$. Fixed cells were washed and blocked by blocking buffer $(0.15 \%(\mathrm{v} / \mathrm{v})$ Triton X-100 and $5 \%(\mathrm{~m} / \mathrm{v})$ BSA in PBS) for $30 \mathrm{~min}$. The dilution buffer of primary antibodies was added. The cells were immunolabeled with the following primary antibody incubated overnight at $4{ }^{\circ} \mathrm{C}$ : rabbit anti-Cyt $\mathrm{C}$ (1:200, \#AF2047, Beyotime, China), mouse anti-TOMM20 (1:150, \#sc-17764, Santa Cruz Bio., USA), mouse anti-flag (1:500, Sigma-Aldrich, USA), rabbit antiBOK (1:200, \#DF3829, Affinity Biosciences, OH, USA), mouse anti-cleaved CASP 3 (1:200, CST, USA) diluted in blocking buffer. The cells were washed with PBS followed by staining with Alexa Fluor 488- (1:1000, Thermo Fisher Scientific, USA), Alexa Fluor 594- (1:1000, Thermo Fisher Scientific, USA) or/ and Alexa Fluor 647-secondary antibody (1:1000, \#AF647, Bioss, Beijing, China) at RT for $1 \mathrm{~h}$. DAPI (Beyotime, China) was used to stain the nuclei. Images were captured using LSM880 Zeiss confocal imaging (63x) system. The co-localization between Cyt $c$ and mitochondria was analyzed with Zeiss Zen Blue software as described previously [24]. A localization index of $100 \%$ indicates that Cyt $c$ and the mitochondria detected by both channels have the same distribution, which is ideal in healthy cells.

The organs or tissue were fixed in $10 \%$ buffered formalin solution, and paraffin sections ( $4 \mu \mathrm{m}$ in thickness) were prepared. Sections were dewaxed and incubated in the pressure boiling citrate buffer solution for $10 \mathrm{~min}$ in stainless steel and was naturally cooled at room temperature. Then the slices were stained followed the above cell immunofluorescence staining method.

\section{Statistical analysis}

Statistical analysis was performed using SPSS version 20.0, and data shown as mean \pm standard derivation (SD). An unpaired student's test was used for two-group comparisons. One-way analyses of variance were used for the analysis of more than two group's comparisons. The normality of the data was confirmed by the Shapiro-Wilk's test and Levene's test. If equal variance were found, the least significant difference test was used; otherwise, the data with the heterogeneity of variance were analyzed using a nonparametric test (Kruskal-Wallis test). 
A

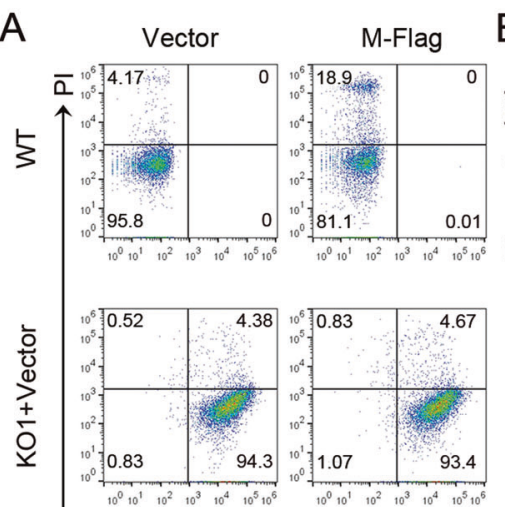

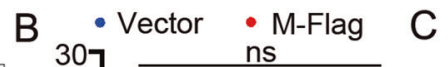

Vector M-Flag

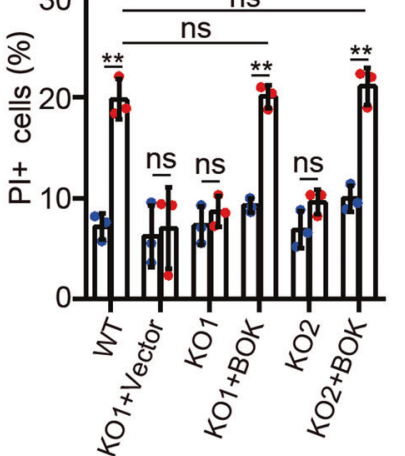

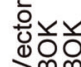

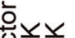

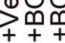

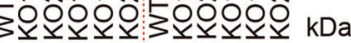

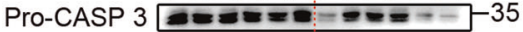

Cleaved CASP 3

Cleaved CASP 9

Cleaved PARP

BOK - $\quad \ldots-\infty \quad \ldots 25$

M-Flag $\square====25$

$\beta$-actin -----------40
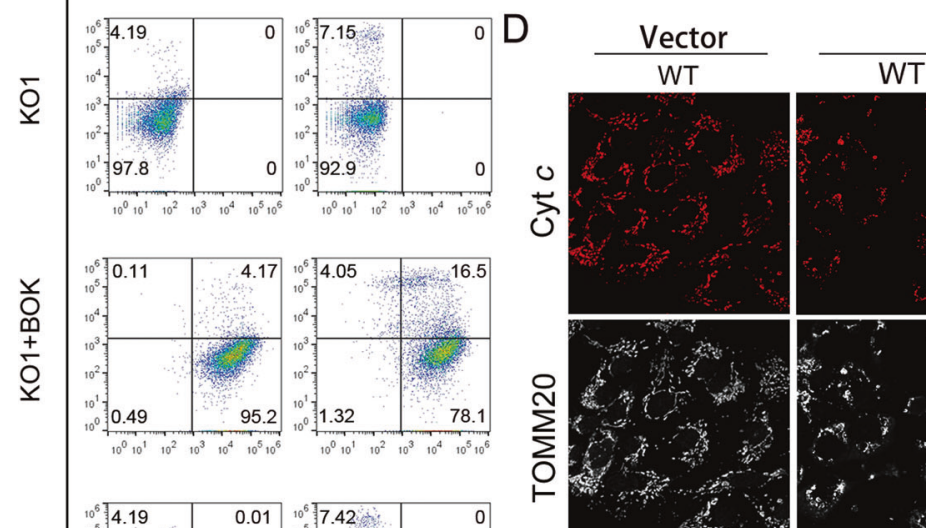

M-Flag

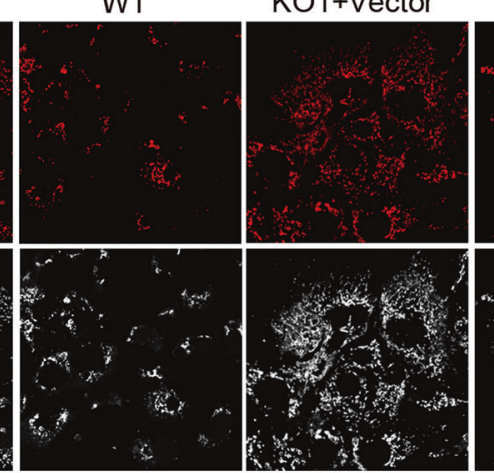

$\mathrm{KO} 1+\mathrm{BOK}$

ญิ
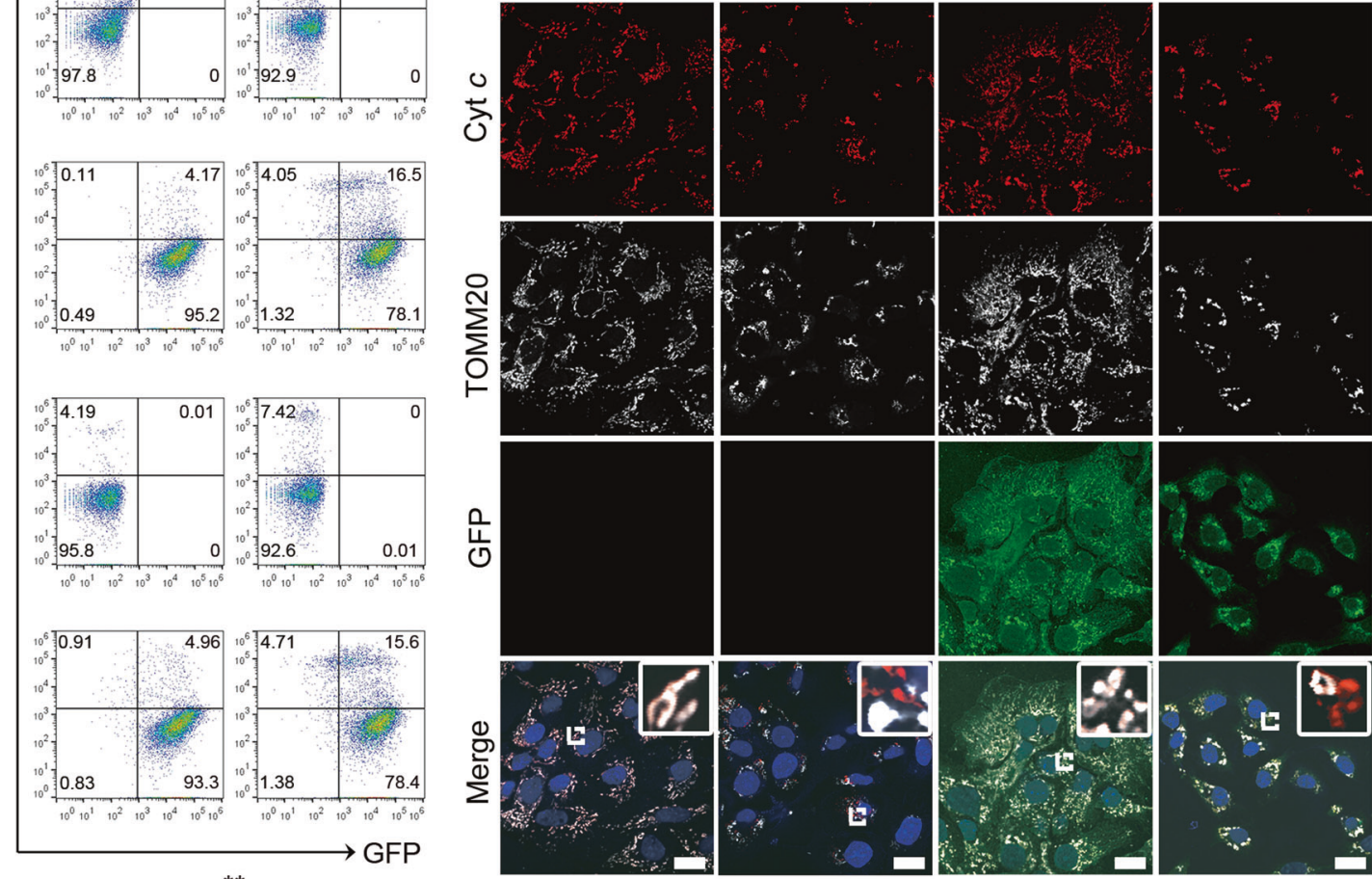

은
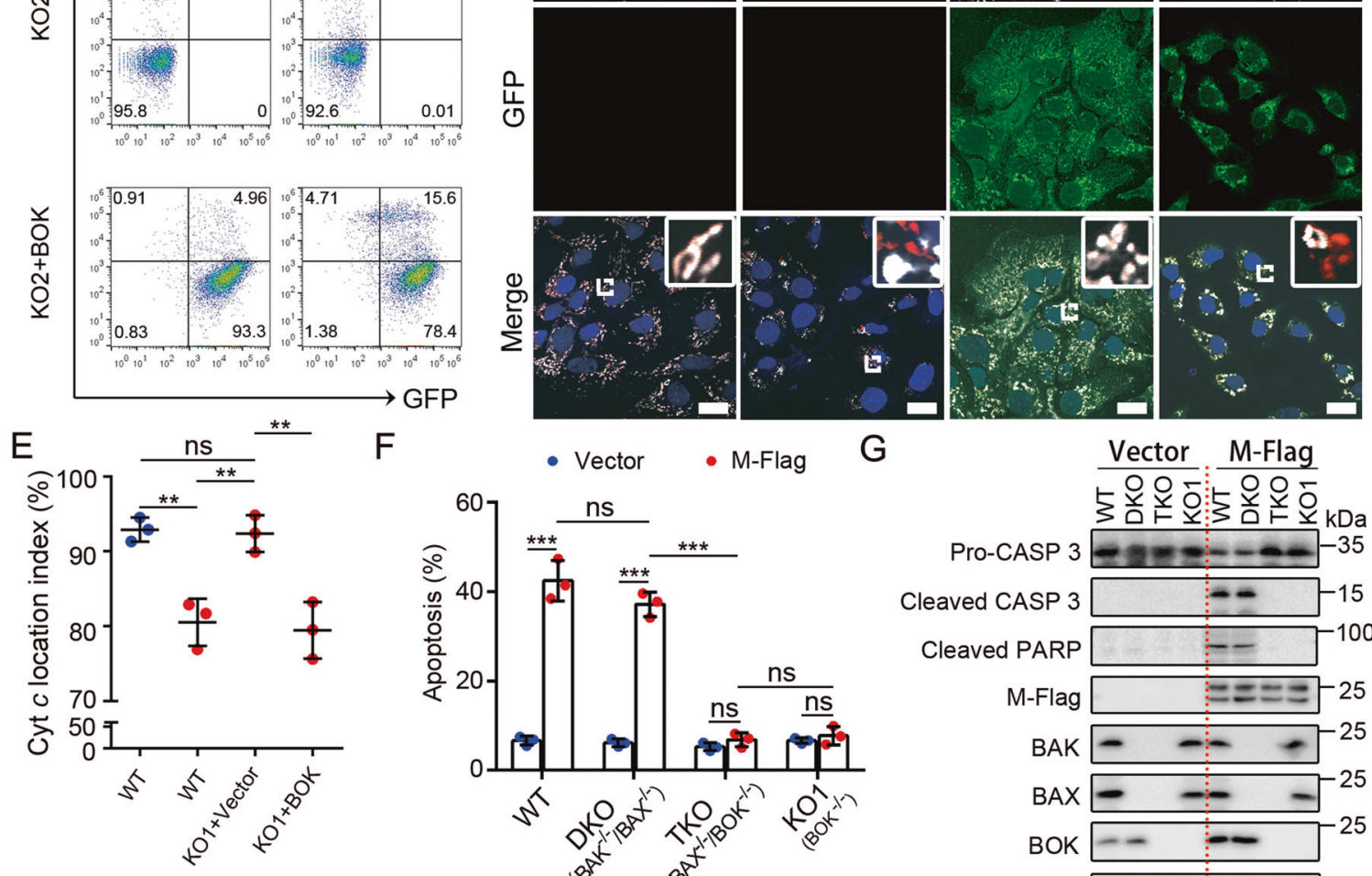

$\mathrm{F}$

G

Vector M-Flag
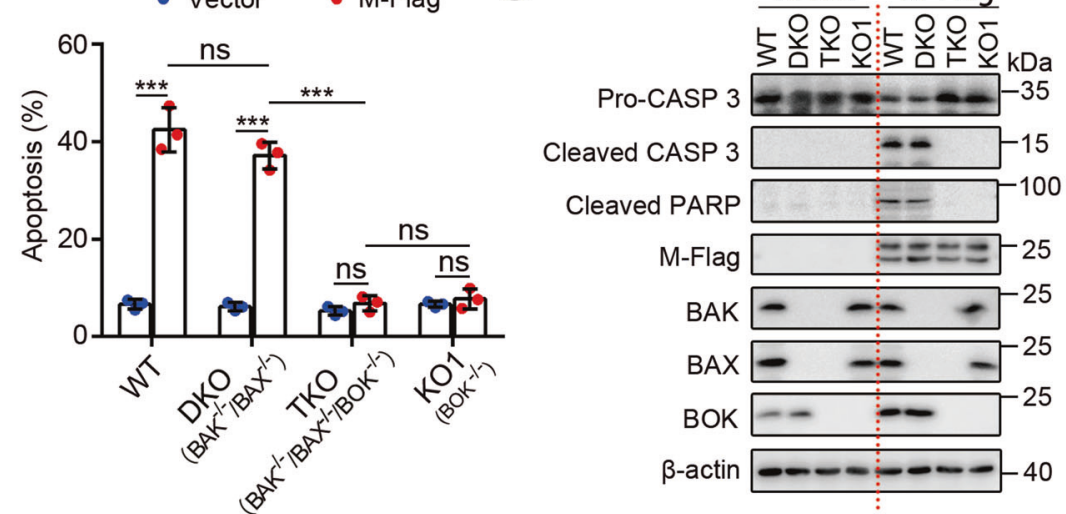

\section{RESULTS}

$M$ protein transient overexpression induced apoptosis

To investigate whether structural proteins of SARS-CoV-2 induce cell apoptosis, H292 and EA.hy926 cells transfected with empty plasmid or plasmids of M-Flag, N-Flag, S-Flag and E-Flag of SARS$\mathrm{CoV}-2$, respectively. The above structural proteins, $M$ protein significantly increased cleaved caspase 3 (CASP 3) activity in both cell lines (Fig. 1A, B). These results indicated that $M$ protein might affect cell apoptosis.

$\mathrm{M}$ protein positive cells showed a nuclear diameter reduction and chromatin condensation compared to control (Fig. 1C, arrows). Furthermore, we observed that the proportion of apoptotic cells was 
Fig. 4 BOK was required for the proapoptotic effect of $\mathbf{M}$ protein overexpression on $\mathbf{H} 292$ derived cells. To determine the role of BOK in the proapoptotic processes of $\mathrm{M}$ protein in $\mathrm{H} 292$ cells, we generated two BOK knockout cell (KO1 and KO2) lines, two corresponding BOK rescued cell (KO1 + BOK and $\mathrm{KO} 2+\mathrm{BOK})$ lines and an empty lentivirus rescued cell (KO + $\mathrm{BOK})$ line. To recover the $\mathrm{BOK}$ expression in the knockout H292-derived cell line, GFP lentivirus vector (pCDH-CMV-MCS-EF1-GFP-T2A-Puro) or carrying BOK was used to transducted the BOK $\mathrm{KO}$ cells. The $\mathrm{PCDH}-\mathrm{CMV}-\mathrm{MCS}-\mathrm{EF} 1-\mathrm{GFP}-\mathrm{T} 2 \mathrm{~A}-\mathrm{Puro}$ cloning and expression lentivirus vector drives expression of Bok from the CMV promoter and drives expression of GFP from EF1 $\alpha$ promoter. A Six H292 derived cell lines, including WT, KO1 + Vector, KO1, KO1 + BOK, KO2 and KO2 + BOK, were transfected with an empty vector (pcDNA6B) (six vertically left panels) or M-flag expressing plasmids (six vertically right panels). After $48 \mathrm{~h}$, the $\mathrm{PI}^{+}$cells were determined by flow cytometry analysis (FACS). B The proportion of $\mathrm{PI}^{+}$cells were further analyzed according to the FACS results in (A). C H292-derived cells were transfected with an empty vector (pCDNA6B) and M-Flag expressing plasmid for $48 \mathrm{~h}$. The Pro-CASP 3, cleaved CASP 3, cleaved CASP 9, cleaved PARP, BOK and M-Flag levels were measured by Western blot. D H292-derived cells were transfected with an empty vector (pcDNA6B) or M-Flag expressing plasmid for $36 \mathrm{~h}$. The Cyt $c$ and TOMM20 were detected by immunofluorescence. The images were acquired using a Zeiss 880 inverted confocal microscope with a $63 \times$ oil-immersion objective. Scale bar, $50 \mu \mathrm{m}$. E Co-localization of mitochondria (TOMM20, white) and Cyt $c$ (red) was analyzed. $n=5-10$ images $/ 63 \times$ fields experiment. Data present

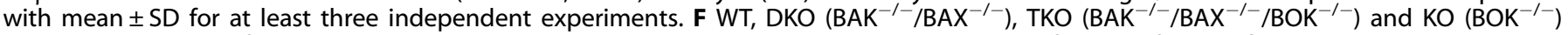
$\mathrm{H} 292$ cells were transfected with an pcDNA6B vector or M-Flag expressing plasmid. At $48 \mathrm{~h}$ after transfection, flow cytometric analysis was used for measuring the proportion of apoptotic cells stained with annexin V-fluorescein 5-isothiocyanate (FITC) and the proportion of apoptotic cells was analyzed. G H292-derived cells (WT, DKO, TKO and KO1) were transfected with an empty vector (pcDNA6B) and M-Flag expressing plasmid for $48 \mathrm{~h}$. The Pro-CASP 3, cleaved CASP 3, cleaved PARP, BAK, BAX, BOK and M-Flag levels were measured by Western blot. Data present with mean \pm SD for three independent experiments. ns non-significant, ${ }^{*} p<0.05 ;{ }^{* *} p<0.01,{ }^{* *} p<0.001$. Data were analyzed with the Kruskal-Wallis test with post hoc Dunn's test.

significantly increased in $\mathrm{M}$ protein overexpressing cells compared to control in both cell lines (Fig. 1D, E).

Real-time morphological observation of H292 and EA.hy926 cells revealed classical apoptosis morphology upon $M$ protein overexpression such as cytoplasm condensation, blebbing of the cell membrane, membrane-bound vesicles formation (Fig. $1 \mathrm{~F}$ and Supplementary Movies S1 and S2). The above data suggested that overexpression of M protein could induce apoptosis in H292 cells and EA.hy926 cells.

\section{Transient overexpression of $M$ protein activated the mitochondrial apoptosis pathway in $\mathrm{H} 292$ cells}

To gain insights into the underlying mechanism of activating CASP 3 by $\mathrm{M}$ protein overexpression in $\mathrm{H} 292$ cells, CASP 8,9 and 12 activity were detected. No alterations in the Fas-associated CASP 8 and endoplasmic reticulum (ED)-involved CASP 12 responses were observed in $M$ protein overexpression cells (Fig. 2A). Notably, the activity of mitochondria-associated CASP 9 was significantly elevated upon $M$ protein overexpression (Fig. 2A). For better identifying the role of CASP played during the $M$ protein proapoptotic process in cells, the CASP 9 specific inhibitor (ZLEHD-FMK) and pan-CASP inhibitor (Q-VD-OPh) were used. ZLEHD-FMK inhibited M protein-induced CASP 3 activation in $\mathrm{H} 292$ cells (Fig. 2B). Further, no difference in the proportion of cleaved CASP 3 positive cells was observed in response to $M$ protein overexpression in the presence of Q-VD-OPh or Z-LEHD-FMK (Fig. 2B, C). These results suggested that apoptosis induced by $M$ protein was CASP 9 dependent.

Irreversible loss of mitochondrial membrane potential $\left(\Delta \Psi_{\mathrm{m}}\right)$ is a significant event in the early apoptotic cells [25]. Immunostaining and FACS results showed that the proportion of cells with low $\Delta \Psi \mathrm{m}$ was higher in the $M$ protein transfection group compared to the control (Fig. 2D-F). A colocalization analysis confirmed the leakage of Cyt $c$ from mitochondria in the M-overexpressing cells (Fig. 2G, H). The above results confirmed that overexpression of $M$ protein activated the mitochondrial apoptosis pathway in $\mathrm{H} 292$ cells.

\section{$M$ protein inhibited the ubiquitination and resulted in cellular accumulation of BOK}

A crucial checkpoint in activating the mitochondrial apoptosis pathway is mitochondrial perforation [17]. To investigate whether $\mathrm{BCL}-2$ family proteins are involved in $\mathrm{M}$ protein induced-apoptosis, we detected the levels of BCL-2, BID, BAK, BAX and BOK in $\mathrm{H} 292$ cells transfected with $M$ protein expressing plasmid. Then, we noticed a significant increase in BOK expression, but no change was found in other proteins (Fig. 3A). $M$ protein induced upregulation of BOK was accompanied by elevating cleaved CASP
3 protein level (Fig. 3B). M protein showed doublet bands (Fig. 3B), which may be related to its O-linked glycosylation [26, 27]. Moreover, BOK redistributed to mitochondria was observed upon $M$ protein overexpression, accompanied by a higher abundance of Cyt $c$ in the cytoplasm (Fig. 3C).

The $M$ protein overexpression had no impact on the transcription level of Bok (Fig. 3D). The BOK degradation rate was slower in $M$ overexpressing cells than in the vector group analyzed by cycloheximide (CHX) chase assay (Fig. 3E). As the ubiquitinproteasome system degrades the BOK [28], we tested whether M protein stabilized BOK by inhibiting its ubiquitination. We immunoprecipitated endogenous BOK with anti-BOK antibody from $\mathrm{H} 292$ cells non-transfected (control) or transfected with an pcDNA6B vector or plasmids encoding Flag-tagged $M$ protein (M-Flag) and analyzed BOK polyubiquitination with both anti-ubiquitin antibody and anti-BOK antibody. Compared with control or vector group, the polyubiquitination of BOK in M-flag group decreased (Fig. 3F, lane 3), indicating that the M-Flag inhibited BOK ubiquitination. Furthermore, the exogenous myc-tagged BOK was immunoprecipitated with anti-myc antibody from 239 T cells in the presence of overexpressed HA-tagged ubiquitin with/without M-Flag overexpressed. Overexpression of $M$ protein made higher level of myctagged BOK be enriched, while the levels of ubiquitinated BOK-Myc were decreased (Fig. 3G). To further investigate how $M$ protein impacted BOK stability, we performed a co-immunoprecipitation (co-IP) assay to evaluate whether M protein interacted with BOK. We found an antibody efficiently precipitated BOK-HA protein against the flag ( $M$ tag), but not by control or SARS-CoV-2 $N$ protein (Fig. 3H). Further, reverse co-IP showed that the $M$ tagged with the flag was precipitated by an antibody against HA (BOK tag) in 293 T cells (Fig. 3I). Next, we used the truncated variants of SARS-CoV-2 $M$ protein (Supplementary Fig. 1) and investigated which region is responsible for binding with BOK. Co-IP results showed that all $M$ protein deletion mutants except for $\triangle T M 1-3$ (endodomain, Fig. $3 \mathrm{~J}$, $\mathrm{K})$, interacted with BOK; thus, the endodomain (ED) of SARS-CoV-2 M protein played a vital role in interacting with BOK. Consistent with the co-IP results, we observed colocalization of SARS-CoV-2 $M$ with $B O K$, and no colocalization of SARS-CoV-2 $\triangle E D$ with BOK (Fig. 3L). These data demonstrated that $M$ protein upregulated the BOK by interacting with BOK via endodomain, and blocking its subsequent degradation.

\section{$M$ protein induced apoptosis required BOK}

To further explore the role of BOK in apoptosis induced by $M$ protein, we used CRISPR/Cas9 to knockout BOK in H292 cells and recombinant exogenous BOK with lentivirus system with GFP reporter gene for further experiments (Supplementary Fig. 2A-C). 


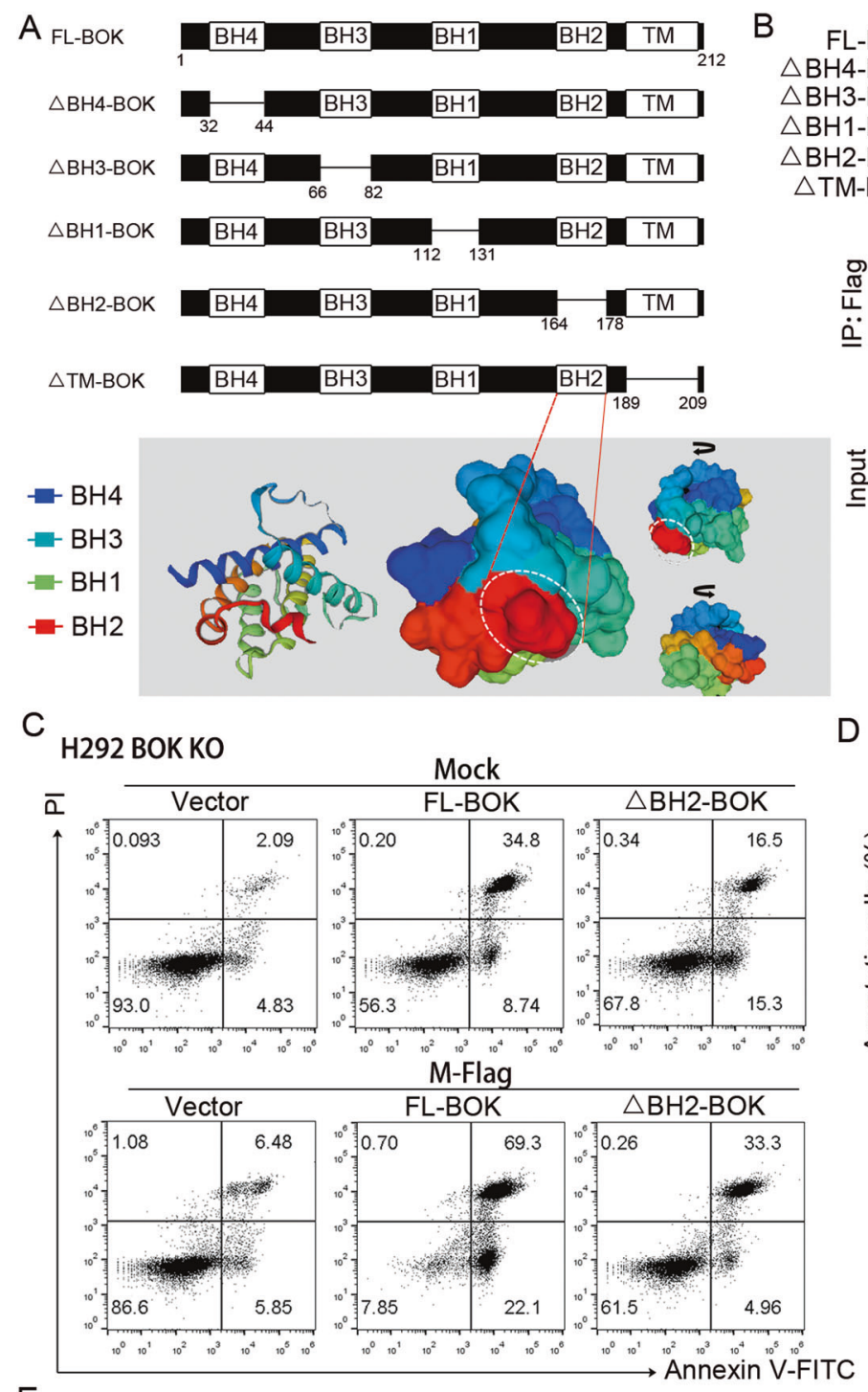

E

H292 BOK KO
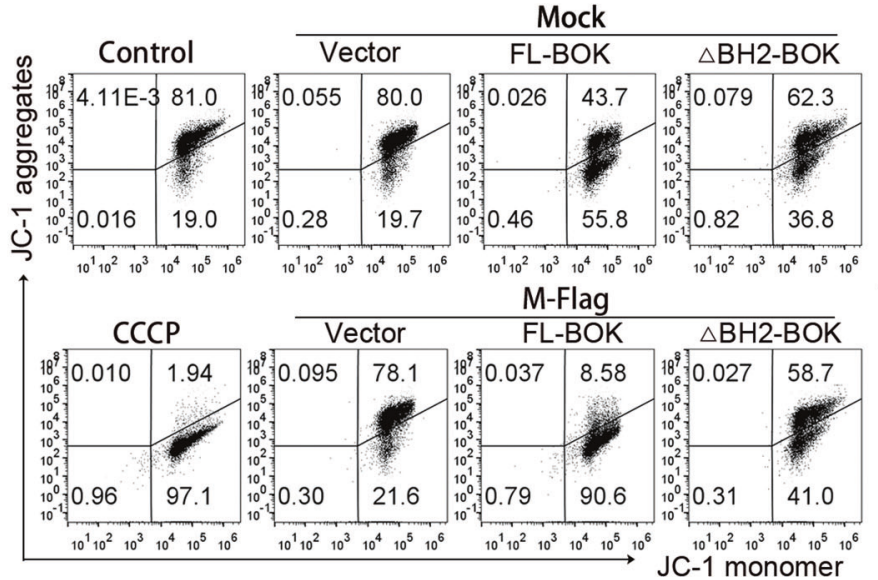
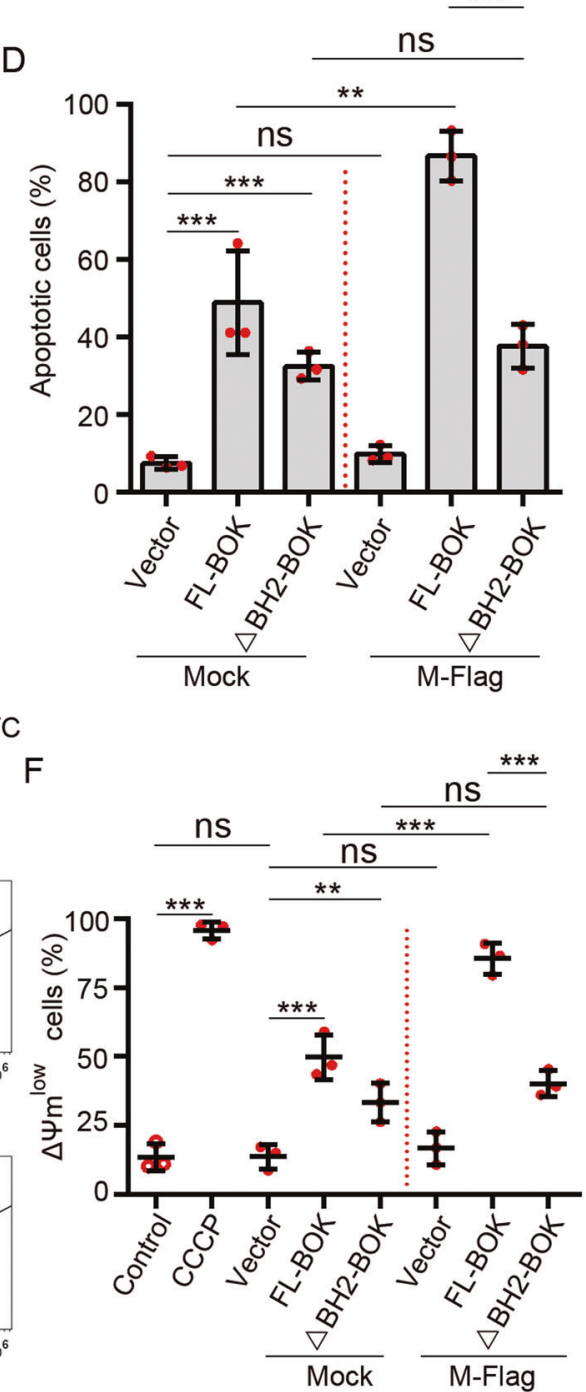

The proportion of $\mathrm{PI}^{+}$cells in wild type (WT), BOK deletion and rescued groups showed no apparent significant difference (Supplementary Fig. 2D, E). Collectively, the results indicated that BOK genetic editing did not impact the viability of $\mathrm{H} 292$ derived cells under normal conditions in vitro.
To further confirm whether $\mathrm{M}$ protein induced mitochondria apoptosis via BOK protein, we used WT and genetically altered H292 cell lines to test their response upon M protein overexpression. The knockout of BOK inhibited apoptosis, prevented cleaved CASP 9, cleaved CASP 3 and cleaved PARP formation, as 
Fig. $5 \mathrm{M}$ protein interacted with BH2 domain and enhanced activity of BOK for inducing apoptosis. A HA-tagged BOK plasmids lacking $\mathrm{BH} 4(\Delta$ from 32 to 44 amino acid residues (aa)), lacking BH3 ( $\triangle$ from 66 to 82 aa), lacking $B H 1$ ( $\triangle$ from 112 to 131 aa), lacking BH2 $(\Delta$ from 164 to $178 \mathrm{aa}$ ) or lacking transmembrane ( $\Delta$ from 189 to $209 \mathrm{aa}$ ) evolved from full length (FL) of BOK and the cartoon representation of the predicted structure of the BOK (BH2 domain was circled by dotted line). B Immunoprecipitation using anti-Flag antibodies from lysate of 293 T cells transfected with distinct HA-tagged BOK plasmids mentioned above and Flag-tagged M. C BOK knockout $\mathrm{H} 292$ derived cells were transfected with an empty vector (pcDNA3.1(+)), FL-BOK, $\triangle \mathrm{BH} 2-\mathrm{BOK}$ alone or cotransfected M-Flag and FL-BOK or $\triangle \mathrm{BH} 2-\mathrm{BOK}$, respectively. Cells were stained with Annexin V/PI after $48 \mathrm{~h}$ of post transfection. D Graph represents result of flow cytometry analysis expressed as histograms of apoptotic cells proportion (Annexin $\mathrm{V}+/ \mathrm{PI}-$ \& Annexin $\mathrm{V}+/ \mathrm{PI}+$ ) under indicated treatments. Data from three separate experiments are presented as mean \pm SD. E BOK knockout $\mathrm{H} 292$ derived cells were transfected with an empty vector (pcDNA3.1 $(+))$, FL-BOK, $\triangle \mathrm{BH} 2-\mathrm{BOK}$ alone or cotransfected $\mathrm{M}$-Flag and FL-BOK or $\triangle \mathrm{BH} 2-\mathrm{BOK}$, respectively. CCCP $(50 \mu \mathrm{M})$ was used as a control for mitochondria depolarization. Cells were stained with JC-1 after $36 \mathrm{~h}$ post transfection. F The quantification of $\Delta \Psi \mathrm{m}$ (only JC-1 monomers staining cells) under indicated treatments. Data from three separate experiments are presented as mean \pm SD. ${ }^{*} p<0.05,{ }^{* *} p<0.01,{ }^{* * *} p<0.001$, ns nonsignificant. Data were analyzed with the Kruskal-Wallis test with post hoc Dunn's test.

well as leakage of Cyt $c$ in $\mathrm{H} 292$ cells upon $\mathrm{M}$ protein overexpression (Fig. 4A-E). We have also confirmed these results by rescue experiments (Fig. 4A-E). Since mitochondria-dependent apoptosis generally needs the presence of $\operatorname{BAK}$ and $\operatorname{BAX}[29,30]$, we generated $\mathrm{BAK}^{-1-} \mathrm{BAX}^{-1-}$ (DKO) and $\mathrm{BAK}^{-1-} \mathrm{BAX}^{-1-} \mathrm{BOK}^{-1-}$ (TKO) $\mathrm{H} 292$ cells to explore whether $M$ protein overexpressioninduced apoptosis requires the BAK and BAX. Similar to WT cells, $M$ protein overexpression-induced cell apoptosis and promoted the cleaved CASP 3 and cleaved PARP expression in DKO cells, but not in the TKO cells and BOK KO1 cells (Fig. 4F, G). The above results indicated that $M$ protein could induce mitochondrial apoptosis pathway via BOK without BAK and BAX.

\section{$M$ protein interacted with $\mathrm{BH} 2$ domain and enhanced the activity of BOK for inducing apoptosis}

To identify the region of BOK protein responsible for interacting with $\mathrm{M}$ protein, five BOK deletion mutants were generated. Co-IP results showed that all $B O K$ protein deletion mutants except for $\triangle \mathrm{BH} 2$, interacted with $\mathrm{M}$ protein (Fig. $5 \mathrm{~A}, \mathrm{~B}$ ). The above results indicated that $\mathrm{M}$ protein interacted with the $\mathrm{BH} 2$ domain of $\mathrm{BOK}$.

To investigate whether the binding of $M$ protein and BOK affected apoptosis, we cotransfected plasmids of M-Flag with FLBOK or $\triangle \mathrm{BH} 2-\mathrm{BOK}$ into $\mathrm{H} 292$ derived cells. Apoptotic and $\Delta \Psi_{\mathrm{m}}{ }^{\text {low }}$ cells proportion was enhanced by overexpression of FL-BOKprotein and $\triangle \mathrm{BH} 2-\mathrm{BOK}$-protein, respectively, compared to control. The percentage of cell apoptosis was markedly enhanced by $M$ cotransfection with FL-BOK but not with $\triangle \mathrm{BH} 2-\mathrm{BOK}$-protein (Fig. $5 \mathrm{C}-\mathrm{F}$ ). These results demonstrated that $\mathrm{M}$ protein bound to the $\mathrm{BH} 2$ domain of $\mathrm{BOK}$ and enhanced activity of $\mathrm{BOK}$ for inducing apoptosis.

\section{Expression of $\mathbf{M}$ protein by lentivirus promoted apoptosis of lung cells and increased the pulmonary permeability in vivo} Next, we investigated the role of $M$ protein in vivo with lentivirus as a carrier (Supplementary Fig. 3). As shown in the Supplementary Fig. 4A, C57BL/6 mice were intratracheally injected with a lentivirus harboring the $M$ and mcherry reporter gene. The mcherry fluorescent signals were detected in the lungs of lentivector group, lenti-M group and lenti-M + Q-VD-OPh mice group, compared to control group (Supplementary Fig. 4B). To assess whether the indicated treatment caused the lung injury, we performed CT examinations of the mice. Intratracheal injection of lentivirus vector produced only minor changes in the lung, compared to control group. The lung presented severe groundglass opacities in lenti-M group (Fig. 6A). The Q-VD-OPh treatment significantly improved pulmonary density compared to those in the lenti-M group (Fig. 6A). Furthermore, we observed the histopathological morphologic alterations of lung tissues. The mcherry $^{+}$cells in the lung sections of lenti-vector group, lenti-M group and lenti-M + Q-VD-OPh group could be observed under the immunofluorescent microscope (Fig. 6B). In the lungs of lenti$M$ group treated mice, we detected a higher number of mcherry ${ }^{+}$ and cleaved CAPS $3^{+}$cells. Furthermore, $M$ protein expression resulted in nucleosome-sized fragments characteristic of apoptosis and being desquamated (Fig. 6B). In addition, $M$ protein expression increased cleaved CASP 9, cleaved CASP 3 and cleaved PARP expression in lung tissues, and this effect was attenuated by intravenous injection of Q-VD-OPh. Furthermore, the level of BOK was significantly increased in lenti-M group (Fig. 6C).

As the cell apoptosis was associated with alteration of pulmonary microvasculature permeability in lung injury [31, 32], we further assessed permeability of the alveolar-capillary barrier in mouse lungs. Expression of M-protein markedly increased the lung wet/dry weight ratio, total cell counts, concentrations of total protein and IgM in the BALF, which was blunted by Q-VD-OPh (Fig. 6D-G). Tracheal injection of PBS and control lentivirus had no or minor alterations on the aforementioned lung edema index. The above data showed that the $M$ protein caused the lung edema and protein infiltration of plasma. Further, we used Evans blue (EB) dye to confirm whether the above phenomenon involved the alveolar-capillary barrier disorders. The result demonstrated that the EB content in lung tissue was markedly higher in the lenti-M group than in other groups (Supplementary Fig. 4C). Q-VD-OPh significantly reduced the amount of EB, indicating less leakage, which was presumably due to less apoptosis (Fig. 6H). Collectively, the CASP associated cell apoptosis triggered by lentivirus expressing $M$ protein induced hyperpermeability in the pulmonary barrier.

\section{Knockdown of BOK suppressed lung edema caused by lentivirus $M$ protein expression in vivo}

To test the role of BOK in pulmonary barrier disorder induced by lentivirus $M$ protein expression in vivo, we used lentivirusmediated short-hairpin RNA (shRNA) targeting BOK. WB results showed that the two BOK shRNA could effectively inhibit the protein level of BOK in mouse lewis lung carcinoma (LLC) cells (Fig. 7A). To further test whether the BOK knockdown altered the cell apoptosis upon expression of M protein, LLC cells were further transducted with lentivirus carrying $M$ protein $(\mathrm{MOI}=15)$ for $72 \mathrm{~h}$ after BOK knockdown. The results showed that the levels of BOK and cleaved CASP 3 were obviously increased after transduction of lentivirus carrying $M$ protein in the PBS and shCtrl. group. On the contrary, the protein level of BOK and cleaved CASP 3 was significantly reduced in the shRNA knockdown groups compared with PBS and the control shRNA group (Fig. 7B).

Next, we performed tracheal injection of a lentivirus expressing either shRNA against BOK or a control without expressing shRNA before treating with lenti-M-mcherry (Fig. 7C). The protein level of cleaved CASP 3, cleaved PARP and BOK in lung tissue was reduced in shBOK1 or shBOK2 group compared to PBS and shCtrl. groups (Fig. 7D). Further, the lung wet/dry ratio, total protein content, IgM concentration and total cell count in the shBOK1group and the shBOK2 group were significantly less than that in the PBS and shCtrl. groups (Fig. 7E-H). These results suggested that the downregulation of BOK could ameliorate pulmonary edema resulted from $M$ protein expression in mice. 

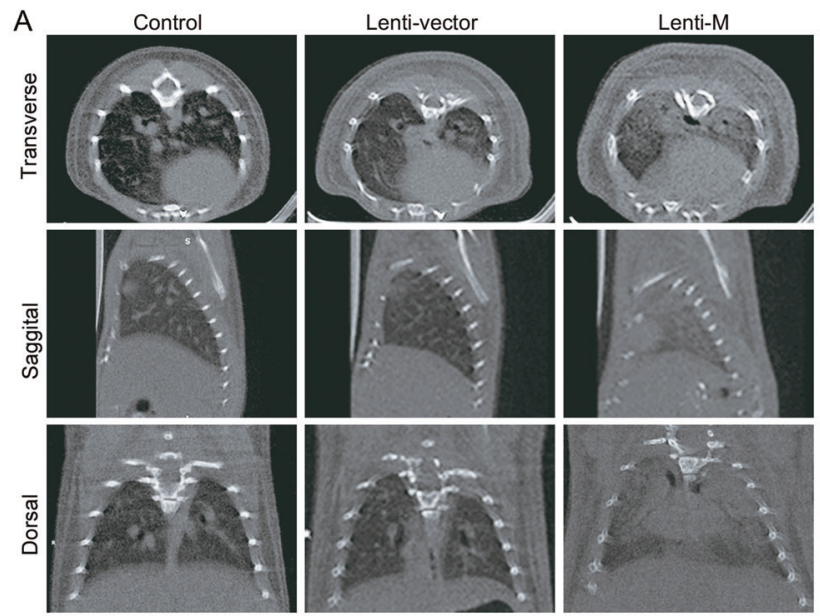

Lenti-M +Q-VD-OPh
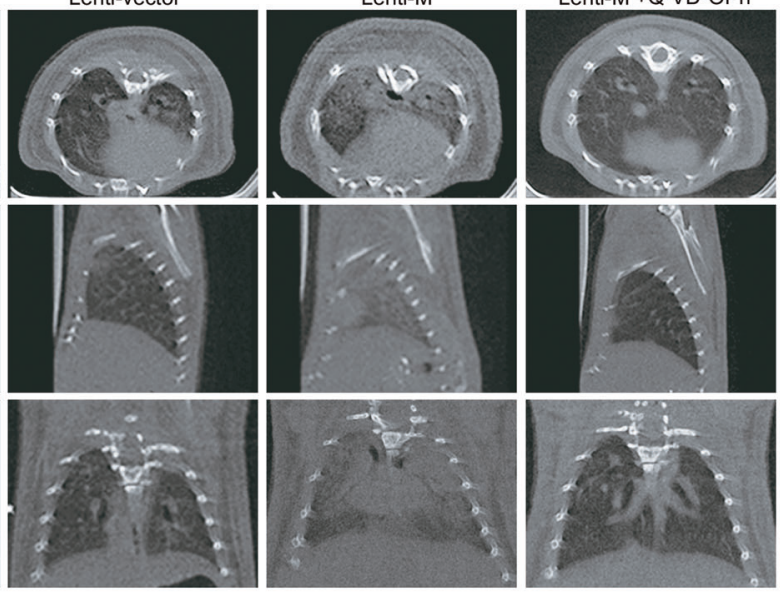

B

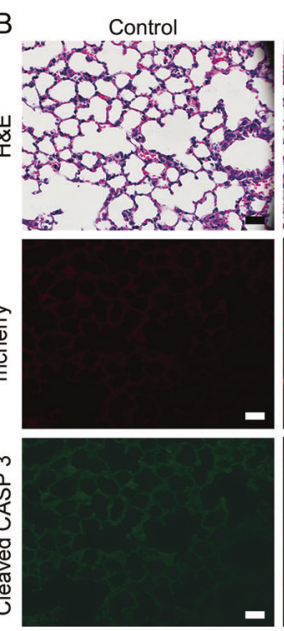

Lenti-vector

Lenti-M
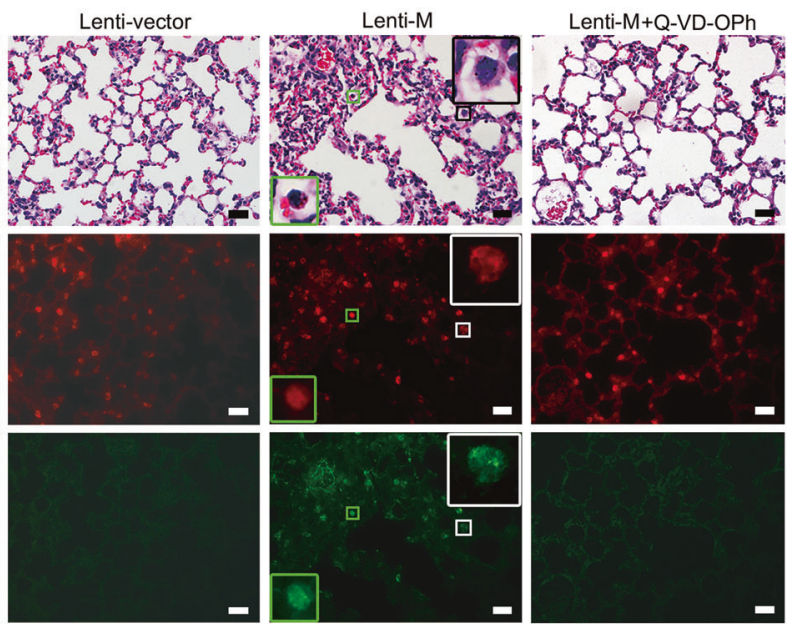

C
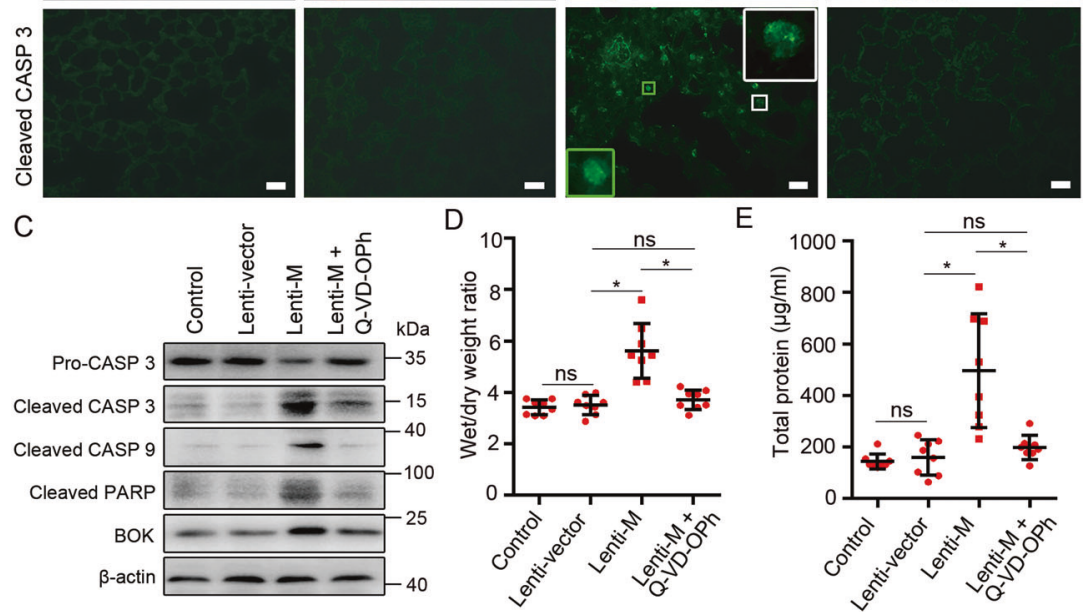

E

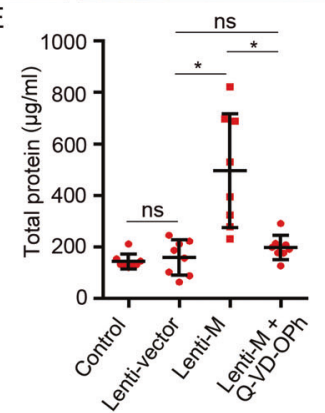

F

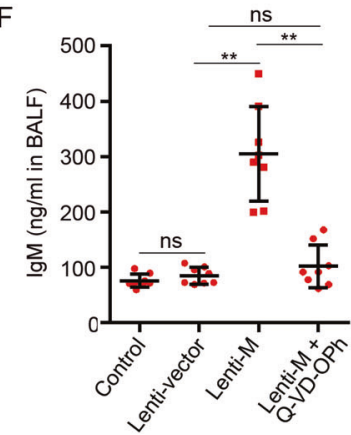

G

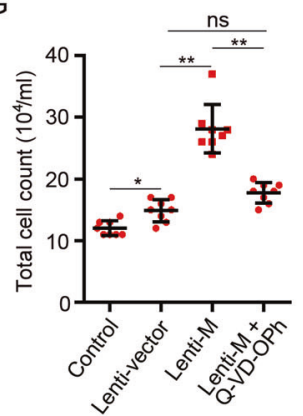

$\mathrm{H}$

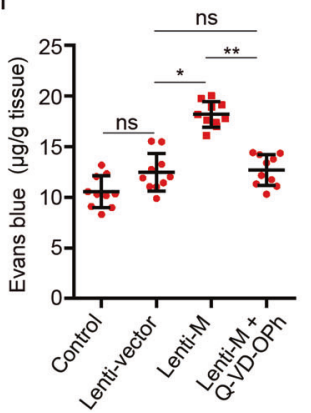

\section{DISCUSSION}

Evidence showed that SARS-CoV-2 could directly induce host cell apoptosis $[10,11,15]$. However, the associated molecular factors involved during cell apoptosis triggered by SARS-CoV-2 are not clear. In this study, we demonstrated that the SARS-CoV-2 M protein induced cell apoptosis via BOK. In vivo results suggested that M-protein expression induced lung cell BOK-associated mitochondrial death, damaged pulmonary barrier integrity and caused lung edema in the mouse.

COVID-19 is characterized by a ground-glass shadow on CT and diffuse alveolar injury [33], suggested that capillary permeable pulmonary edema is a driving factor for respiratory failure. 
Fig. 6 M protein expression destroyed pulmonary barrier integrity in vivo. C57BL/ 6 mice were randomly divided into four groups ( $n=8$ mice per group) for intratracheally injection with PBS (Control), Lenti-vector (lentivirus carrying empty vector), Lenti-M (lentivirus carrying SARS-CoV-2 M protein) and Lenti-M + Q-VD-OPh (pan CASP inhibitor), respectively. A Computed tomography (CT) comparison of mouse lungs after three days of post treatments. B Histopathology and immunofluorescence of representative lung tissue sections stained with hematoxylin and eosin or anti-cleaved CASP 3. The images were taken using the Leica DM5500 microscope (20x). Scale bar: $20 \mu \mathrm{m}$. C Immunoblotting analysis was performed on lung tissue samples. D Lung wet-to-dry weight ratio in the lungs. $\mathbf{E}$, $\mathbf{F}$ Concentrations of total protein (E) and IgM (F) (large size protein in plasma, $900 \mathrm{kDa}$ ) in bronchoalveolar lavage fluid (BALF) of the groups. G Total cell counts in BALF of the groups. H Quantification of Evans blue contents in the lung tissue from the groups $(n=10$ mice per group). All values are means \pm SD. ${ }^{*} p<0.05,{ }^{* *} p<0.01$, ns non-significant. Data were analyzed with the Kruskal-Wallis test with post hoc Dunn's test.

A

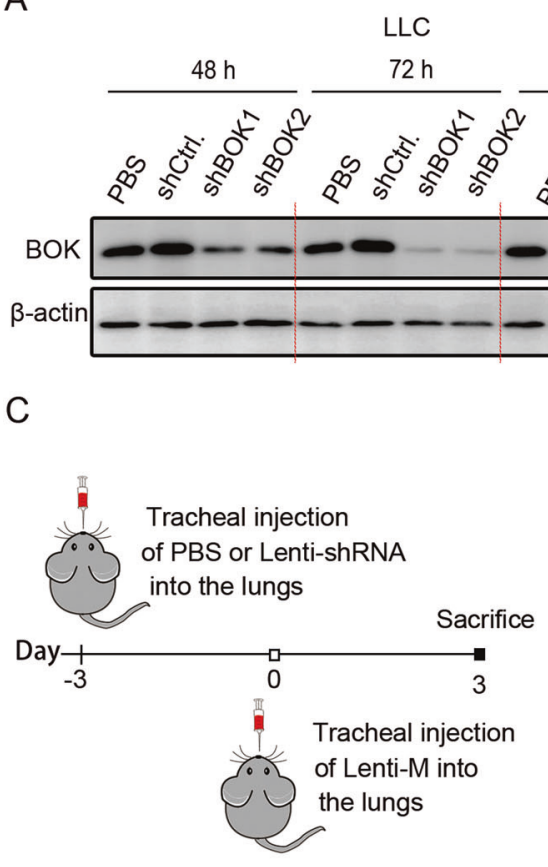

B

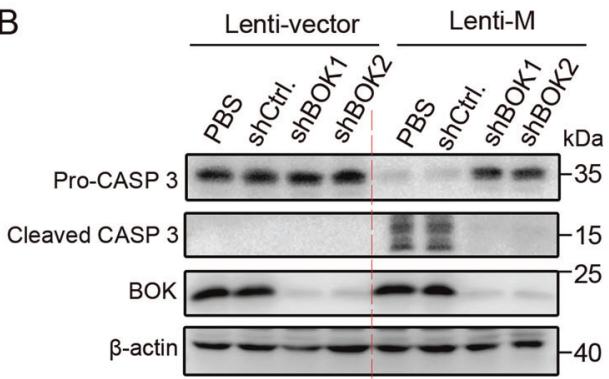

D
$96 \mathrm{~h}$

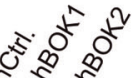

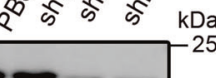

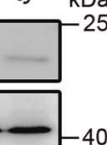

$\mathrm{E}$
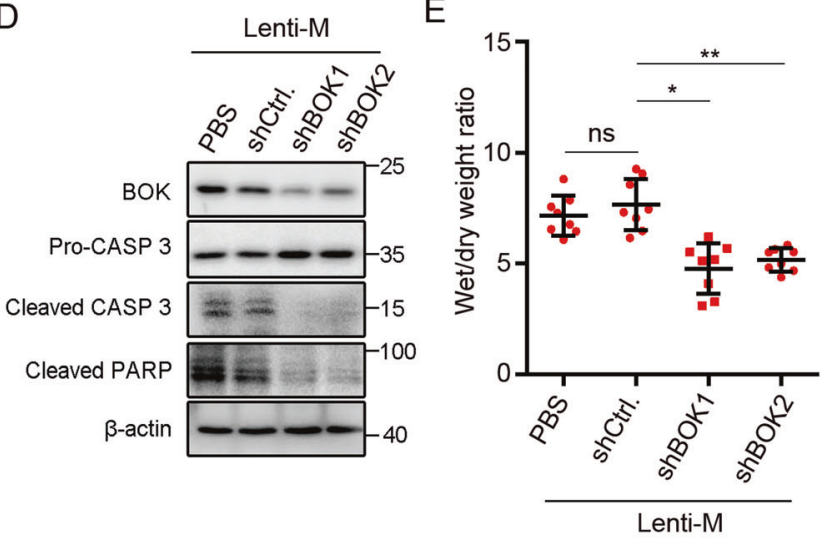

$\mathrm{F}$

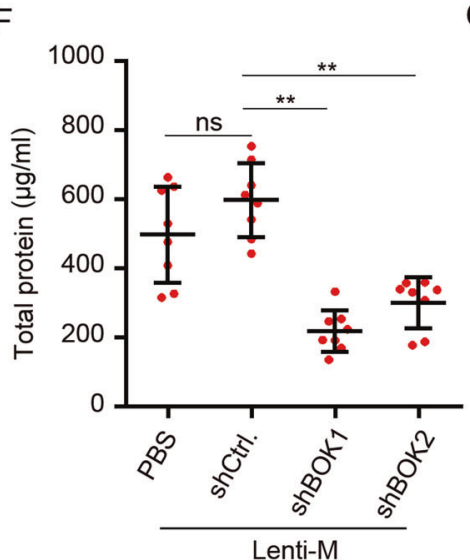

G

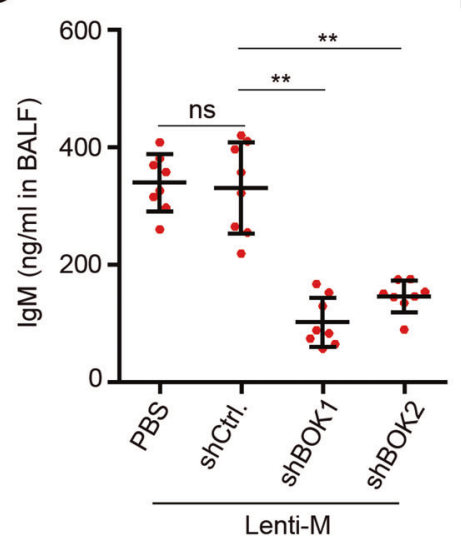

$\mathrm{H}$

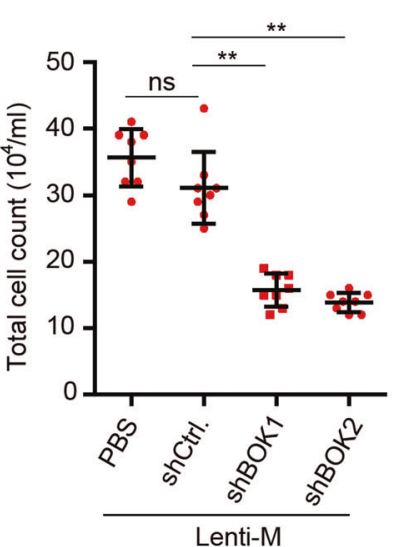

Fig. 7 BOK knockdown alleviated lung edema induced by the $\mathbf{M}$ protein expression. C57BL/6 mice were subjected to nontransducted (PBS), transducted with negative control shRNA (shCtrl.), anti-BOK-shRNA1(shBOK1), and anti-BOK-shRNA2 (shBOK2) tracheal injection for three days, and then injected with lentivirus expressing SARS-CoV-2 M protein for three days ( $n=8$ per group). A Western blot analysis of BOK protein levels in mouse lewis lung carcinoma (LLC) cells PBS, shCtrl lentivirus, or shBOK1, and shBOK2 group for $48 \mathrm{~h}, 72 \mathrm{~h}$, and $96 \mathrm{~h}$, respectively. B Western blot analysis of Pro-CASP 3, cleaved CASP 3 and BOK protein levels in LLC derived cells upon transduction with lentivector or lenti-M for $72 \mathrm{~h}$. C Schematic representation of the experimental process. D Representative immunoblotting analysis of Pro-CASP 3 , cleaved CASP 3, BOK and cleaved PARP protein levels in the lungs. $\mathbf{E}$ Lung wet-to-dry weight ratio in the groups. $\mathbf{F}$ Total protein concentration in the BALF. G IgM concentration was measured in the indicated groups. $\mathbf{H}$ Total cell count in the BALF. All values are means \pm SD. ${ }^{*} p<0.05$, ${ }^{* *} p<0.01$, ns non-significant. Data were analyzed with the Kruskal-Wallis test with post hoc Dunn's test. 
Consistent with this view, the autopsy results show that patients who died due to COVID-19 have severe lung epithelial and endothelial damage $[3,34]$. The pathogenesis of this manifestation and molecular mechanisms remain unclear. As an important part of the alveolar-capillary barrier, the epithelial layer prevents pulmonary edema $[35,36]$. In the current study, in vitro studies revealed that the SARS-CoV-2 M protein had a direct proapoptotic effect on the human lung epithelial cells and umbilical vein cells. The detrimental effect of $M$ protein on lung tissue was confirmed in in vivo experiments and caused serum $\lg M$ and albumin leakage into the BALF. Since the SARS-CoV-2 virus particles can be observed in bronchiolar and alveolar epithelial cells of COVID-19 patients [37], the $M$ protein may be one of the detrimental factors aggravating lung injury during COVID-19.

From the aspects of similar sequence and structure to $B A X$ and BAK, BOK is classified into "killers" effector subgroup of the BCL-2 family [17]. BOK overexpression triggers intrinsic apoptosis [38]. The role and function of BOK have been mainly investigated in the development and progression of cancer [17]. In healthy cells, BOK are mainly localized to and partially inserted into the membranes of the Golgi apparatus as well as the ER and associated membranes [39]. BOK can directly mediate mitochondria membrane permeabilization, which is independent of the participation of other proteins in vitro [40]. In host cells, BOK is degraded after ubiquitination modification [28]. Once the degradation pathway is impeded, the stabilized BOK protein perturbs mitochondrial membrane permeability [40]. In our study, we observed the colocalization of BOK with M protein with immunofluorescence in 293 T cells. Since the SARSCoV-2 $\mathrm{M}$ protein is primarily localized to the ER and Golgi $[19,41]$, we conjectured that $M$ protein stabilized BOK by inhibiting its ubiquitination-degradation at the ER. Previous studies reported that BOK activity can be regulated by activated BID or anti-apoptotic BCL-2 proteins through BH3-BOK domain $[28,42]$. Here, $M$ protein enhanced the proapoptotic activity of $\mathrm{BOK}$ through its $\mathrm{BH} 2$ domain. Deletion of $\mathrm{BH} 2$ domain inhibited, but not abolished, the proapoptotic ability of BOK overexpression in the $\mathrm{H} 292$ cells. $\mathrm{BH} 2$ domain of BOK may not be required, but as a regulated domain, for its function as an initiator of cell death, which is similar with BAX [43]. Similar to the proapoptotic effect of BOK showed in our study, BOK knockdown ovarian carcinoma cells were resistant to drug-induced cell death (taxol, cisplatin, camptothecin) [44]. These findings contradict the study that BOK protects lung cells from cadmium chlorideinduced cell death [45]. The discrepancy may be attributed to the cell-intrinsic differences (e.g., expression of endogenous $\mathrm{BOK}$, cell lines) and requires further investigation. In addition, BOK knockdown could blunt the lung cell apoptosis and subsequent lung edema induced by $M$ protein expression. Overall, these findings demonstrated the proapoptotic function of $\mathrm{BOK}$ in the lentivirus carrying $M$ protein infection mouse model.

In summary, we demonstrate that BOK is critical for SARS-CoV-2 $M$ protein-mediated apoptosis. By suppressing the CASP activity or downregulating the level of BOK, cell death and tissue disorders could be alleviated in the lungs of mice infected with lentivirus carrying $M$ protein. These findings may serve not only to elucidate the mechanisms underlying SARS-CoV-2 associated lung edema in COVID-19 patient, but also to provide a potential target for the development of therapeutic strategies for the amelioration of SARS-CoV-2 induced lung and vascular injury.

\section{DATA AVAILABILITY STATEMENT}

The datasets used and/or analyzed during the current study are available from the corresponding author on reasonable request.

\section{REFERENCES}

1. Xu Z, Shi L, Wang Y, Zhang J, Huang L, Zhang C, et al. Lancet Respir Med. 2020;8:420-22.

2. Goshua G, Pine AB, Meizlish ML, Chang $C H$, Zhang $H$, Bahel $P$, et al. Endotheliopathy in COVID-19-associated coagulopathy: evidence from a single-centre, cross-sectional study. Lancet Haematol. 2020;7:e575-82.

3. Matthay MA, Leligdowicz A, Liu KD. Biological mechanisms of COVID-19 acute respiratory distress syndrome. Am J Respir Crit Care Med. 2020;202:1489-91.

4. Galluzzi L, Vitale I, Aaronson SA, Abrams JM, Adam D, Agostinis P, et al. Molecular mechanisms of cell death: recommendations of the Nomenclature Committee on Cell Death 2018. Cell Death Differ. 2018;25:486-541.

5. Danthi P. Viruses and the diversity of cell death. Annu Rev Virol. 2016;3:533-53.

6. Fung TS, Liu DX. Human coronavirus: host-pathogen interaction. Annu Rev Microbiol. 2019;73:529-57.

7. Zhang J, Han Y, Shi H, Chen J, Zhang X, Wang X, et al. Swine acute diarrhea syndrome coronavirus-induced apoptosis is caspase- and cyclophilin D- dependent. Emerg Microbes Infect. 2020;9:439-56.

8. Colmenero I, Santonja C, Alonso-Riano M, Noguera-Morel L, Hernandez-Martin A, Andina D, et al. SARS-CoV-2 endothelial infection causes COVID-19 chilblains: histopathological, immunohistochemical and ultrastructural study of seven paediatric cases. Br J Dermatol. 2020;183:729-37.

9. Katsura $H$, Sontake V, Tata A, Kobayashi $Y$, Edwards CE, Heaton BE, et al. Human lung stem cell-based alveolospheres provide insights into SARS-CoV-2-mediated interferon responses and pneumocyte dysfunction. Cell Stem Cell. 2020;27:890-904.

10. Bao L, Deng W, Huang B, Gao H, Liu J, Ren L, et al. The pathogenicity of SARSCoV-2 in hACE2 transgenic mice. Nature.2020;583:830-3.

11. Chan JF, Zhang AJ, Yuan S, Poon VK, Chan CC, Lee AC, et al. Simulation of the clinical and pathological manifestations of coronavirus disease 2019 (COVID-19) in a golden syrian hamster model: implications for disease pathogenesis and transmissibility. Clin Infect Dis. 2020;71:2428-46.

12. Jiang RD, Liu MQ, Chen Y, Shan C, Zhou YW, Shen XR, et al. Pathogenesis of SARSCoV-2 in transgenic mice expressing human angiotensin-converting enzyme 2. Cell. 2020;182:50-8.

13. Lin $P$, Wang $M$, Wei $Y$, Kim $T$, Wei $X$. Coronavirus in human diseases: mechanisms and advances in clinical treatment. MedComm (Beijing). 2020;1:270-301.

14. Lai CW, Chan ZR, Yang DG, Lo WH, Lai YK, Chang MD, et al. Accelerated induction of apoptosis in insect cells by baculovirus-expressed SARS-CoV membrane protein. FEBS Lett. 2006;580:3829-34.

15. Padhan $K$, Minakshi $R$, Towheed MAB, Jameel $S$. Severe acute respiratory syndrome coronavirus $3 a$ protein activates the mitochondrial death pathway through p38 MAP kinase activation. J Gen Virol. 2008;89:1960-9.

16. Hekman RM, Hume AJ, Goel RK, Abo KM, Huang J, Blum BC, et al. Actionable cytopathogenic host responses of human alveolar type 2 cells to SARS-CoV-2. Mol Cell. 2021;81:212.

17. Moldoveanu T, Czabotar PE. BAX, BAK, and BOK: a coming of age for the BCL-2 family effector proteins. Cold Spring Harb Perspect Biol. 2020;12:a036319.

18. Zhang J, Cruz-Cosme R, Zhuang MW, Liu D, Liu Y, Teng S, et al. A systemic and molecular study of subcellular localization of SARS-CoV-2 proteins. Signal Transduct Target Ther. 2020;5:269.

19. Zheng $Y$, Zhuang MW, Han L, Zhang J, Nan ML, Zhan $P$, et al. Severe acute respiratory syndrome coronavirus 2 (SARS-CoV-2) membrane (M) protein inhibits type I and III interferon production by targeting RIG-I/MDA-5 signaling. Signal Transduct Target Ther. 2020;5:299.

20. Neupane AS, Willson M, Chojnacki AK, Vargas ESCF, Morehouse C, Carestia A, et al. Patrolling alveolar macrophages conceal bacteria from the immune system to maintain homeostasis. Cell. 2020;183:110-25.

21. Bierman A, Yerrapureddy A, Reddy NM, Hassoun PM, Reddy SP. Epidermal growth factor receptor (EGFR) regulates mechanical ventilation-induced lung injury in mice. Transl Res. 2008;152:265-72.

22. Xu M, Wang F, Li G, Wang X, Fang $X$, Jin H, et al. MED12 exerts an emerging role in actin-mediated cytokinesis via LIMK2/cofilin pathway in NSCLC. Mol Cancer. 2019;18:93.

23. Bi HL, Zhang XL, Zhang YL, Xie X, Xia YL, Du J, et al. The deubiquitinase UCHL1 regulates cardiac hypertrophy by stabilizing epidermal growth factor receptor. Sci Adv. 2020;6:eaax4826.

24. Yang Y, Kuang L, Li L, Wu Y, Zhong B, Huang X. Distinct mitochondria-mediated Tcell apoptosis responses in children and adults with coronavirus disease 2019 . J Infect Dis. 2021;224:1333-44.

25. Zamzami N, Marchetti P, Castedo M, Zanin C, Vayssiere JL, Petit PX, et al. Reduction in mitochondrial potential constitutes an early irreversible step of programmed lymphocyte death in vivo. J Exp Med. 1995;181:1661-72.

26. Dawood AA. Glycosylation, ligand binding sites and antigenic variations between membrane glycoprotein of COVID-19 and related coronaviruses. Vacunas.2021;22:1-9. 
27. Snijder EJ, Bredenbeek PJ, Dobbe JC, Thiel V, Ziebuhr J, Poon LL, et al. Unique and conserved features of genome and proteome of SARS-coronavirus, an early splitoff from the coronavirus group 2 lineage. J Mol Biol. 2003;331:991-1004.

28. Llambi F, Wang YM, Victor B, Yang M, Schneider DM, Gingras S, et al. BOK is a non-canonical BCL-2 family effector of apoptosis regulated by ER-associated degradation. Cell. 2016;165:421-33.

29. Lindsten T, Ross AJ, King A, Zong WX, Rathmell JC, Shiels HA, et al. The combined functions of proapoptotic Bcl-2 family members bak and bax are essential for normal development of multiple tissues. Mol Cell. 2000;6:1389-99.

30. Wei MC, Zong WX, Cheng EH, Lindsten T, Panoutsakopoulou V, Ross AJ, et al. Proapoptotic BAX and BAK: a requisite gateway to mitochondrial dysfunction and death. Science. 2001;292:727-30.

31. Kushimoto S, Taira Y, Kitazawa Y, Okuchi K, Sakamoto T, Ishikura H, et al. The clinical usefulness of extravascular lung water and pulmonary vascular permeability index to diagnose and characterize pulmonary edema: a prospective multicenter study on the quantitative differential diagnostic definition for acute lung injury/acute respiratory distress syndrome. Crit Care. 2012;16:R232.

32. Villar J, Zhang $H$, Slutsky AS. Lung repair and regeneration in ARDS: role of PECAM1 and Wnt signaling. Chest. 2019;155:587-94.

33. Michalick L, Weidenfeld S, Grimmer B, Fatykhova D, Solymosi PD, Behrens F, et al. Plasma mediators in patients with severe COVID-19 cause lung endothelial barrier failure. Eur Respir J. 2021;57:2002384.

34. Varga Z, Flammer AJ, Steiger $P$, Haberecker M, Andermatt R, Zinkernagel AS, et al. Endothelial cell infection and endotheliitis in COVID-19. Lancet. 2020;395:1417-8.

35. Herzog EL, Van Arnam J, Hu B, Krause DS. Threshold of lung injury required for the appearance of marrow-derived lung epithelia. Stem Cells. 2006;24:1986-92.

36. Tan KS, Lim RL, Liu J, Ong HH, Tan VJ, Lim HF, et al. Respiratory viral infections in exacerbation of chronic airway inflammatory diseases: novel mechanisms and insights from the upper airway epithelium. Front Cell Dev Biol. 2020;8:99.

37. Yao XH, He ZC, Li TY, Zhang HR, Wang Y, Mou H, et al. Pathological evidence for residual SARS-CoV-2 in pulmonary tissues of a ready-for-discharge patient. Cell Res. 2020;30:541-3.

38. Stehle D, Grimm M, Einsele-Scholz S, Ladwig F, Johanning J, Fischer G, et al. Contribution of $\mathrm{BH} 3-$ domain and transmembrane-domain to the activity and interaction of the pore-forming Bcl-2 Proteins Bok, Bak, and Bax. Sci Rep. 2018;8:12434.

39. Echeverry N, Bachmann D, Ke F, Strasser A, Simon HU, Kaufmann T. Intracellular localization of the BCL-2 family member BOK and functional implications. Cell Death Differ. 2013;20:785-99.

40. Zheng JH, Grace CR, Guibao CD, McNamara DE, Llambi F, Wang YM, et al. Intrinsic instability of BOK enables membrane permeabilization in apoptosis. Cell Rep. 2018;23:2083-94.

41. Gordon DE, Jang GM, Bouhaddou M, Xu J, Obernier K, O'Meara MJ, et al. A SARSCoV-2-human protein-protein interaction map reveals drug targets and potential drug-repurposing. bioRxiv. 2020. Preprint.

42. Fernandez-Marrero Y, Bleicken S, Das KK, Bachmann D, Kaufmann T, Garcia-Saez AJ. The membrane activity of BOK involves formation of large, stable toroidal pores and is promoted by CBID. FEBS J. 2017;284:711-24.

43. Zha H, Aime-Sempe $C$, Sato T, Reed JC. Proapoptotic protein Bax heterodimerizes with $\mathrm{Bcl}-2$ and homodimerizes with Bax via a novel domain (BH3) distinct from BH1 and BH2. J Biol Chem. 1996;271:7440-4.

44. Einsele-Scholz S, Malmsheimer S, Bertram K, Stehle D, Johanning J, Manz M, et al. Bok is a genuine multi-BH-domain protein that triggers apoptosis in the absence of Bax and Bak. J Cell Sci. 2016;129:3054.
45. Zhang F, Ren L, Zhou S, Duan P, Xue J, Chen H, et al. Role of B-Cell Lymphoma 2 Ovarian Killer (BOK) in acute toxicity of human lung epithelial cells caused by cadmium chloride. Med Sci Monit. 2019;25:5356-68.

\section{ACKNOWLEDGEMENTS}

We thank Dr. Li Huiyan for providing plentiCRISPR v2 and pLVX-nCoV-M-mCherry.

\section{AUTHOR CONTRIBUTIONS}

YY, WYJ, and $H X$ conceptualized study. WYJ, YY, MXJ and WZY collected, analyzed the data and edited the manuscript. All co-authors made comments to the manuscript.

\section{FUNDING}

This work was supported by grants from the National Natural Science Foundation of China (31470877), the National Science and Technology Key Projects for Major Infectious Diseases (2017ZX10302301-002), the Support Scheme of Guangzhou for Leading Talents in Innovation and Entrepreneurship (No.2017004), the Guangdong Scientific and Technological Research Project for Prevention and Treatment of COVID-19 (2020A111128022, 2020B111112003), the Guangdong Scientific and Technological Research Special Fund for COVID-19 (202020012612200001), the Zhuhai Scientific and Technological Research Project for COVID-19 containment (ZH22036302200029PWC), the Zhuhai Industrial Technological Research and Development Project for Prevention and Treatment of COVID-19 (ZH22046301200018PWC), the Three Major Scientific Research Projects of Sun Yatsen University (20200326236) (X.H.), the Science and Technology Planning Project of Guangzhou (201704020226 and 201604020006), and Development Project of Foshan Fourth People's Hospital (FSSYKF-2020003, FSSYKF-2020017).

\section{COMPETING INTERESTS}

The authors declare no competing interests.

\section{ETHICS APPROVAL}

Our studies did not include human participants, human data or human tissue. The animal studies were approved by the Ethics Committee of Sun-Yat Sen University (No.00109), in accordance with UK Animals (Scientific Procedures) Act 1986.

\section{ADDITIONAL INFORMATION}

Supplementary information The online version contains supplementary material available at https://doi.org/10.1038/s41418-022-00928-x.

Correspondence and requests for materials should be addressed to Xi Huang.

Reprints and permission information is available at http://www.nature.com/ reprints

Publisher's note Springer Nature remains neutral with regard to jurisdictional claims in published maps and institutional affiliations. 